\title{
Validating the BISON Fuel Performance Code to Integral LWR Experiments
}

\author{
R. L. Williamson*, K. A. Gamble, D. M. Perez, S. R. Novascone, G. Pastore, R. J. Gardner, J. D. Hales \\ Fuel Modeling and Simulation, Idaho National Laboratory, P.O. Box 1625, Idaho Falls, ID 83415-3840
}

W. Liu, A. Mai

ANATECH Corporation, 5435 Oberlin Dr., San Diego, CA 92121

\begin{abstract}
BISON is a modern finite element-based nuclear fuel performance code that has been under development at Idaho National Laboratory (INL) since 2009. The code is applicable to both steady and transient fuel behavior and has been used to analyze a variety of fuel forms in 1D spherical, 2D axisymmetric, or 3D geometries. Code validation is underway and is the subject of this study. A brief overview of BISON's computational framework, governing equations, and general material and behavioral models is provided. BISON code and solution verification procedures are described, followed by a summary of the experimental data used to date for validation of Light Water Reactor (LWR) fuel. Validation comparisons focus on fuel centerline temperature, fission gas release, and rod diameter both before and following fuel-clad mechanical contact. Comparisons for 35 LWR rods are consolidated to provide an overall view of how the code is predicting physical behavior, with a few select validation cases discussed in greater detail. Results demonstrate that 1) fuel centerline temperature comparisons through all phases of fuel life are very reasonable with deviations between predictions and experimental data within $\pm 10 \%$ for early life through high burnup fuel and only slightly out of these bounds for power ramp experiments, 2) accuracy in predicting fission gas release appears to be consistent with state-of-the-art modeling and with the involved uncertainties and 3) comparison of rod diameter results indicates a tendency to overpredict clad diameter reduction early in life, when clad creepdown dominates, and more significantly overpredict the diameter increase late in life, when fuel expansion controls the mechanical response. Initial rod diameter comparisons are not satisfactory and have led to consideration of additional separate effects experiments to better understand and predict clad and fuel mechanical behavior. Results from this study are being used to define priorities for ongoing code development and validation activities.
\end{abstract}

Keywords: validation, verification, BISON

\section{Introduction}

Since 2009, Idaho National Laboratory (INL) has been developing next-generation capabilities to model nuclear fuel, resulting in the BISON code [1, 2]. BISON is a parallel, finite element-based tool that solves the coupled non-linear partial differential equations associated with nuclear fuel behavior. BISON supports the use of one-, two-, and three-dimensional meshes and uses implicit time integration, important for the widely varied time scales in nuclear fuel simulation. A software architecture is employed which minimizes the programming required to add new features such as material and behavior models.

BISON is intended to be a multi-fuels code. Though primarily applied to LWR fuel to date, the code has been used to analyze TRISO-coated particle fuel [2] and metal fuel in rod

\footnotetext{
${ }^{*}$ Corresponding author

Email addresses: Richard.Williamsoneinl.gov (R. L. Williamson), Kyle.Gambleeinl.gov (K. A. Gamble), Danielle.Perezeinl.gov (D. M. Perez), Stephen. Novascone@inl.gov (S. R. Novascone),

Giovanni.Pastoreeinl.gov (G. Pastore), Russell.Gardnereinl.gov (R. J. Gardner), Jason. Hales@inl.gov (J. D. Hales),

Wenfeng.Liu@anatech.com (W. Liu), Anh.Mai@anatech.com (A. Mai)
}

and plate form [3, 4], design and interpret fuel irradiation experiments [5] and investigate novel fuel concepts [6].

The simulation of strongly coupled multiphysics phenomena such as nuclear fuel behavior is a challenging computational undertaking, with any simulation involving the interaction of several submodels describing specific phenomena [7]. To ensure that the complexity of the simulation is not masking errors in one or more submodels, and to assess software reliability and numerical methods, code and solution verification is needed. From the beginning, the development of BISON and related software has been accompanied by the creation of numerous verification tests that are run every time the code is modified, laying the foundation for subsequent validation. A recent paper outlines how this has been implemented in BISON [8].

In order to assess the fidelity of BISON's physical modeling for LWR fuel rods, validation efforts are underway. The validation base for LWR fuel considered here includes 35 rods, covering different reactor operating conditions and burnup ranges. BISON predictions are assessed against experimental data including thermal behavior early in life, thermal and fission gas release behavior throughout base irradiation and during power 
ramping, and mechanical behavior via rod diameter comparisons following both base irradiation and power ramps.

Validation of other LWR fuel performance codes with capability similar to BISON have been reported in the past. Examples include ENIGMA [9], FALCON [10], TRANSURANUS [11] and FRAPCON [12]. For each of these codes, validation includes consideration of thermal, fission gas, and mechanical behavior, in a similar manner as reported herein for BISON. In addition, references [10, 12] include clad oxidation and [10] includes accident behavior, which are all ongoing validation efforts with BISON. Note that for TRANSURANUS, validation of individual material models is also described in [11]. Similar separate effects validation is ongoing with BISON and will be reported elsewhere.

The paper begins with a brief overview of BISON's computational framework, governing equations, and general material and behavioral models. BISON code and solution verification procedures are then described, followed by a summary of the experimental data used for LWR validation. In each results section, comparisons of measurements and predictions are consolidated to provide an overall view of how the code is predicting physical behavior, with a few select validation cases discussed in greater detail.

\section{BISON Background}

\subsection{Computational framework}

BISON is built upon INL's Multiphysics Object-Oriented Simulation Environment (MOOSE) [13], a parallel, finite elementbased framework to solve systems of coupled non-linear partial differential equations using the Jacobian-free Newton Krylov (JFNK) method [14]. This enables investigation of computationally large problems, for example a full stack of discrete pellets in a LWR fuel rod, or every rod in a full reactor core.

\subsection{Governing equations and general constitutive behavior}

The BISON governing relations currently consist of fullycoupled partial differential equations for energy, species, and momentum conservation [1]. Users can select a subset of these equations (e.g., energy and momentum for thermomechanics analysis) within the input file. The code employs both nonlinear kinematics, which accounts for large deformation, and nonlinear material behavior. A detailed description of the nonlinear kinematics is provided in [1]. Nonlinear material behavior includes temperature dependent thermal and mechanical properties as well as instantaneous plasticity and creep. For plasticity and creep models, strains are calculated implicitly utilizing the radial return method. The specific procedure used in BISON is outlined in [15].

\subsection{Material and behavioral models}

BISON is a general fuels performance code with capabilities for modeling LWR fuel rods, TRISO fuel particles and metallic fuel. This paper focuses on validation of BISON against integral LWR fuel experiments that contain $\mathrm{UO}_{2}$ fuel and Zircaloy cladding. BISON separates the material properties and behavior into three distinct categories: the fuel, the cladding, and the fuel-to-clad gap. Basic descriptions of the models incorporated into BISON to predict LWR fuel performance are outlined below. Additional details can be found in the BISON theory manual [16].

\subsection{1. $\mathrm{UO}_{2}$ fuel}

BISON includes empirical models to describe temperature and burnup dependent thermal conductivity, temperature dependent specific heat, isotropic solid and gaseous fission product swelling, densification, fracture via relocation, and fission gas production and release. BISON contains thermal and irradiation creep models for $\mathrm{UO}_{2}$, however, in the absence of a robust smeared cracking model to relieve the stresses experienced in the fuel, the creep rate predicted is unrealistically high. Therefore, in comparing to the experimental cases in this work the mechanical behavior of the fuel was treated as elastic. In addition, a model based on $[17,18]$ was used to capture the radial power factor throughout the fuel due to the formation of the high plutonium content rim region during irradiation.

In this study the fuel thermal conductivity is calculated using the (Nuclear Fuel Industry Research) NFIR model proposed by Marion [19], which contains a temperature dependent term that accounts for self-annealing of the fuel as the temperature increases. Solid swelling is linearly proportional to the burnup and is computed using the model outlined in MATPRO [20]. Fission gas release (FGR) is computed by a physics-based model that draws on the approach described in [21]. While retaining a level of complexity suitable for application to engineering scale fuel analysis and consistent with the uncertainties pertaining to some parameters, the model incorporates a direct description of the fundamental physical processes of gas generation, diffusion and precipitation in grains, growth and coalescence of gas bubbles at grain faces, and thermal gas release. Fission gas release and gaseous swelling are modeled as inherently coupled. This model also captures the rapid fission gas release kinetics (burst release) during transients [22]. Fuel densification early in life, which is due to an annealing process under high temperatures and pressures that causes some of the fabrication porosity to be removed, is captured using a modified version of the ESCORE model suggested by Rashid et al. [23]. To account for fuel cracking and pellet-fragment relocation prior to contact, the empirical ESCORE relocation model [24] is used; this model was calibrated by Swiler et al. [25] for use in BISON.

\subsubsection{Zircaloy Cladding}

For the validation cases considered in this study the cladding material is one of two zirconium-based alloys: Zircaloy-2 (BWR) or Zircaloy-4 (PWR). Models exist for temperature dependent thermal conductivity and specific heat, thermal and irradiation creep, instantaneous plasticity and irradiation growth. For the cases considered in this work, the clad remained near normal operating temperatures; thus, the thermal conductivity was assumed to be constant at $16.0 \mathrm{~W} / \mathrm{m}-\mathrm{K}$. The Zircaloy thermal and irradiation creep models available in BISON were given by 
Limbäck and Andersson [26] and depend upon the microstructure formed during manufacturing. Model coefficients vary depending on if the cladding is stress relieved (SRA), partiallyrecrystallized (PRXA), or fully recrystallized (RXA). The SRA model applies to either Zircaloy-2 or Zircaloy-4, whereas the PRXA and RXA models apply to Zircaloy-2 only. In this work all experimental cases investigated were of the SRA type. In cases where cladding stress exceeds the yield strength, an instantaneous plasticity model can be applied. The model assumes linear strain hardening beyond the yield point. In this study only one case (Risø-3 GE7 power ramp) experienced cladding stress above yield, resulting in a peak plastic strain of roughly $1 \%$. For small plastic strains such as this, a linear strain hardening approximation is very reasonable. Irradiation growth occurs in zirconium-based alloys due to the hexagonal-closed-packed (HCP) crystal structure and results in anisotropic mechanical behavior. The irradiation growth model used was developed by Franklin [27].

\subsubsection{Fuel-to-clad gap}

Accurate simulation of the evolution of the fuel-to-clad gap is important since the gap behavior has a substantial effect on fuel temperature and cladding mechanical behavior. The BISON mechanical contact model is based on the methodology of Heinstein and Laursen [28], which utilizes node to face constraints to prevent nodes on one surface from penetrating the face of another surface. The evolution of the gap width is determined using a geometric search algorithm. Gap heat transfer is modeled in the traditional manner with the total conductance across the gap computed as a sum of the gas conductance, the increased conductance due to solid-solid contact, and the conductance due to radiant heat transfer [29]. Jump distances are determined using the Kennard model [30]. The BISON defaults for fuel and cladding roughnesses are 2 and $1 \mu \mathrm{m}$, respectively. While these values may seem small they have a significant impact on temperature predictions as the gap closes and are representative of the roughnesses that can be obtained during manufacture of the fuel and cladding. These values are used unless the experimental data provides additional information.

For LWR fuel, the pressure in the gap and plenum is computed assuming a single cavity volume and using the ideal gas law. The moles of gas, the temperature, and the cavity volume are free to change with time. The moles of gas at any time is computed as the original amount of gas (computed based on original pressure, temperature, and volume) plus the amount in the cavity due to fission gas released. The gas temperature is computed based on the fuel surface and cladding interior temperatures. The cavity volume is computed based on the evolving pellet and cladding geometry.

\section{BISON Verification}

Essentially, verification ensures that each piece of code performs as expected and is the foundation upon which a strong validation basis is built. To that end, considerable emphasis has been placed on verification in BISON. Referencing Roache
[31], Oberkampf [32] points out that verification has two components, referred to as code and solution verification. Strongly influenced by Oberkampf's work, in this paper we refer to code verification as the mathematical correctness of the code implementation and solution verification as a quantitative assessment of the numerical accuracy of a given solution to a particular problem.

\subsection{Code Verification}

Paraphrasing earlier work by Hales et al. [8], code verification is the process of determining whether a software implementation of a mathematical model accurately represents that model. By this definition, it is most applicable to individual modules or submodels in an overall system model. With code verification showing that each submodel calculates the correct result for a representative set of mathematically-conceived problems, the code can be used with confidence as the validation work begins.

BISON includes an extensive set of code verification problems, from fundamental finite element solid mechanics and heat transfer tests, to problems specific to nuclear fuel models. Each of these verification problems is small and runs quickly. This being the case, it is practical to run them all with every code change. Doing this minimizes the likelihood of unintended consequences of any code change and guarantees that all of the verification problems are running properly at all times. BISON code verification is described in detail in [8].

\subsection{Solution Verification}

To quantitatively evaluate the numerical accuracy of BISON validation problems, simulations with representative validationproblem features were spatially and temporally resolved and the results compared. These representative features are: loading (i.e., power supplied to the fuel, coolant pressure on the cladding), boundary conditions, geometry, mesh, and material models. A typical power history was assumed as shown in Figure 1. Note that this power history is not a discrete series of powers for specified durations, as may be common for older fuel performance codes. It is, rather, a continuous function of time. The boundary conditions that represent coolant temperature and pressure were typical for a pressurized water reactor.

The spatial resolution simulations consisted of four levels of mesh refinement, which are summarized in Table 1. Figure 2 shows a mesh with refinement level $\mathrm{C}$, which is the typical mesh density used in BISON LWR validation work.

Table 1: The number and aspect ratios of the elements used in the various meshes in the solution verification study.

\begin{tabular}{lcccc} 
Mesh & A & B & C & D \\
\hline No. Axial Elements Fuel & 275 & 550 & 550 & 1375 \\
No. Radial Elements Fuel & 3 & 6 & 12 & 22 \\
No. Axial Elements Clad & 281 & 556 & 556 & 813 \\
No. Radial Elements Clad & 1 & 3 & 4 & 5 \\
Aspect Ratio Fuel & 9.85 & 9.85 & 19.70 & 14.45 \\
Aspect Ratio Clad & 22.19 & 33.29 & 44.38 & 37.53 \\
\hline
\end{tabular}




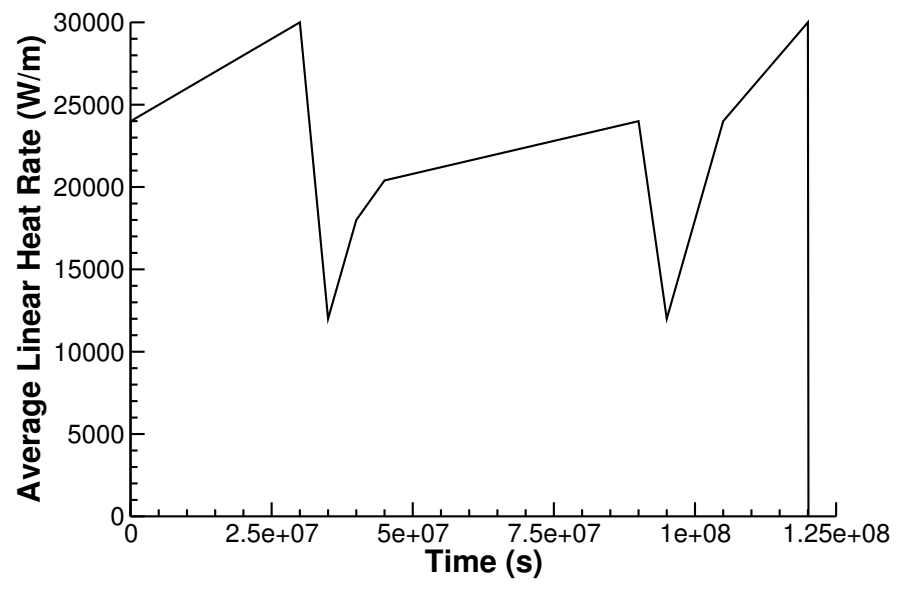

Figure 1: Representative power history used for the spatial and temporal solution verification studies.

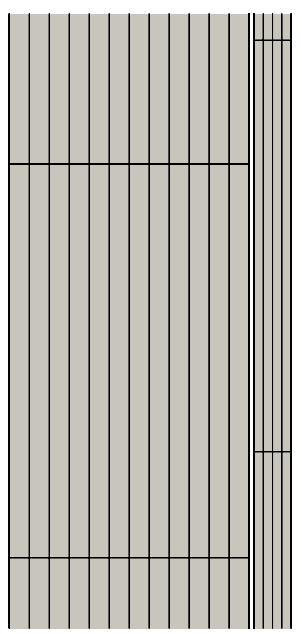

Figure 2: Illustration of a section of mesh $\mathrm{C}$ showing the radial and axial element sizes. Mesh $\mathrm{C}$ is representative of the mesh used for all validation cases used in this study.

The figures of merit in this resolution study are parameters that are important to all validation simulations, which are: power, fuel centerline temperature (FCT), fission gas release (FGR), and rod diameter. As defined here, power is the total nuclear power obtained by integrating the volumetric fission rate over the entire fuel volume. Figure 3 shows relative error calculations from these figures of merit, where results from refinement level D are the basis for relative error calculations. For the parameters of interest, the estimated spatial error using the typical validation mesh (Mesh C) is on the order of $1 \%$ or less, which is adequate for validation studies. Note that power has a larger relative error than the other quantities considered. Power is more sensitive to mesh refinement due to the steep gradient in the radial power profile near the pellet periphery.

Figure 4 shows results from the temporal resolution study. The mesh with refinement level $\mathrm{C}$ was used for these calculations, where the number of times steps was increased and the relative error calculation is with respect to the simulation with the greatest number of times steps. The acceptable number of time steps was determined by decreasing time step size (i.e. in-

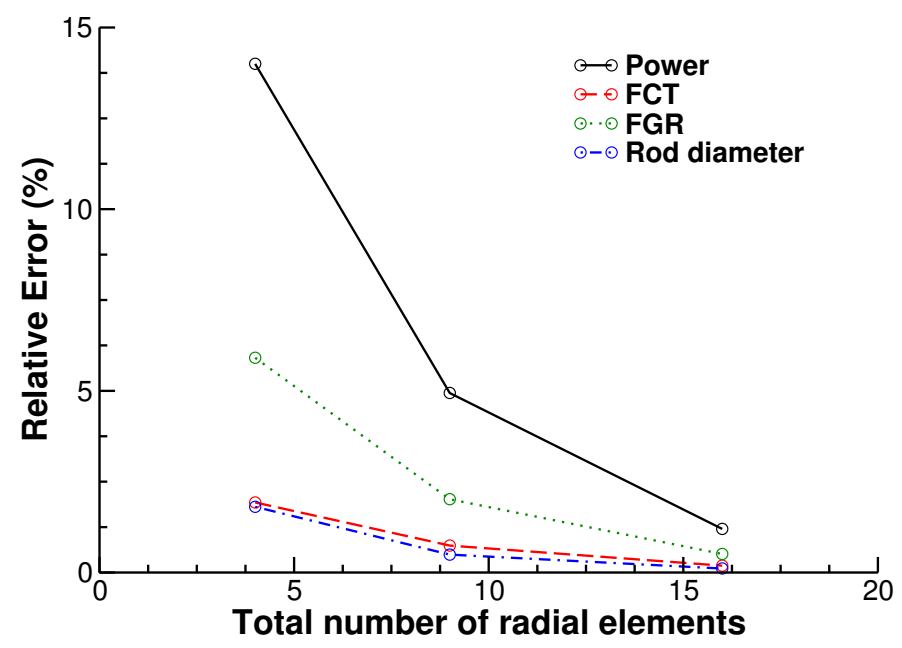

Figure 3: Spatial resolution results for the solution verification study. Relative percent error is presented for four metrics of interest: Power, FCT, FGR, and rod diameter as a function of the total number of radial elements in the mesh. The relative error is with respect to the finest mesh studied, Mesh D.

creasing the total number of time steps) and observing the relative error and the change in relative error. In this case, the relative error is very small even for the lowest number of time steps. Therefore, time resolution is adequate in all cases.

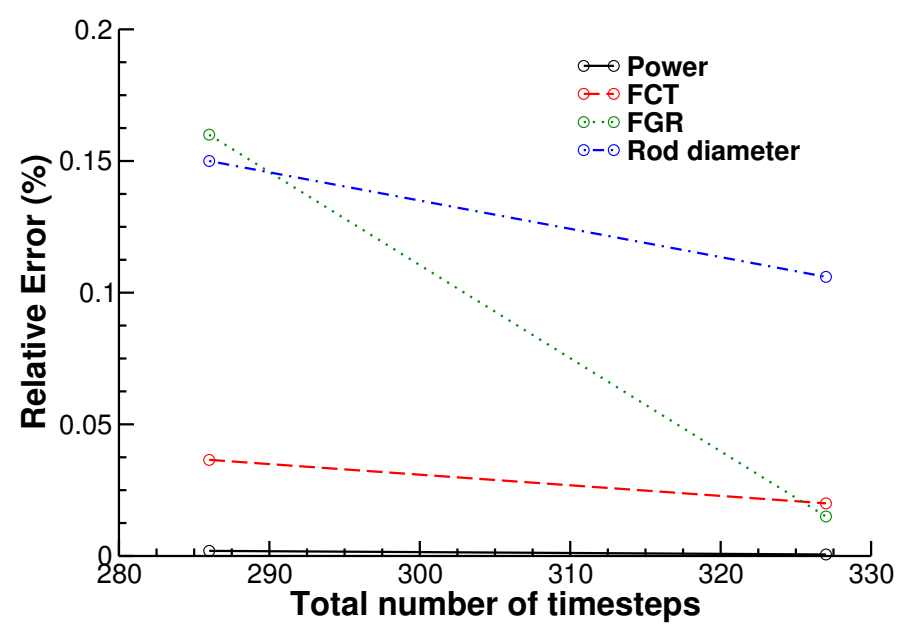

Figure 4: Temporal resolution results for the solution verification study. Relative percent error is presented for four metrics of interest: Power, FCT, FGR, and Rod diameter as a function of the total number time steps taken in the simulation. The relative error is with respect to the case with the most time steps, 681.

\section{Experimental Data Used for Validation}

Table 2 summarizes the experimental fuel rods that have been used for BISON LWR validation and are listed by experiment name, rod designation, final rod-averaged burnup, and measured quantity. These quantities include fuel centerline temperature (FCT), fission gas release (FGR), and cladding outer diameter (Rod Dia) both before and following fuel-clad mechanical contact. Many of these integral rod experiments were 
chosen based on INL participation in the IAEA sponsored FUMEX- 5.2. Validation of Thermal Behavior

III Coordinated Research Project and are priority cases from either FUMEX-II [33] or FUMEX-III [34]. The experiments considered to date include pure $\mathrm{UO}_{2}$ fuel. Future BISON developments will involve code extension to the analysis of LWR MOX and Gd-doped $\mathrm{UO}_{2}$ fuel as well.

Table 2: Overview of the main integral experimental data used for validation of BISON.

\begin{tabular}{|c|c|c|c|c|c|c|}
\hline Experiment & Rod & $\begin{array}{c}\text { Final } \\
\text { Burnup } \\
(\mathrm{MWd} / \mathrm{kgU})\end{array}$ & FCT & FGR & $\begin{array}{l}\text { Rod } \\
\text { Dia }\end{array}$ & Ref \\
\hline IFA-431 & $1 *$ & $\approx 4$ & $\mathrm{X}$ & & & [35] \\
\hline IFA-431 & $2 *$ & $\approx 4$ & $\mathrm{X}$ & & & [35] \\
\hline IFA-431 & $3 *$ & $\approx 4$ & $\mathrm{X}$ & & & [35] \\
\hline IFA-431 (3D) & $4 *$ & $\approx 4$ & $\mathrm{X}$ & & & {$[35,36]$} \\
\hline IFA-432 & $1 *$ & $\approx 32$ & $\mathrm{X}$ & & & {$[35,37]$} \\
\hline IFA-432 & $2^{*}$ & $\approx 32$ & $\mathrm{X}$ & & & {$[35,37]$} \\
\hline IFA-432 & $3^{*}$ & $\approx 32$ & $\mathrm{X}$ & & & {$[35,37]$} \\
\hline IFA-515.10 & A1** & 86.6 & $\mathrm{X}$ & & & {$[38]$} \\
\hline IFA-534 & 18 & 59.0 & & $X$ & & [37] \\
\hline IFA-534 & 19 & 59.0 & & $\mathrm{X}$ & & [37] \\
\hline IFA-535 & 809 & 54.4 & & $\mathrm{X}$ & & [37] \\
\hline IFA-535 & 810 & 54.4 & & $\mathrm{X}$ & & [37] \\
\hline IFA-562.2 & 15 & 56.7 & $\mathrm{X}$ & $\mathrm{X}$ & & [39] \\
\hline IFA-562.2 & 16 & 56.2 & $\mathrm{X}$ & $\mathrm{X}$ & & [39] \\
\hline IFA-562.2 & 17 & 56.2 & $\mathrm{X}$ & $\mathrm{X}$ & & [39] \\
\hline IFA-597.3 & 8 & 68.1 & $\mathrm{X}$ & $\mathrm{X}$ & & [37] \\
\hline Ris $\varnothing-2$ & GE-m & 15.8 & & $\mathrm{X}$ & $\mathrm{X}$ & [37] \\
\hline Ris $\varnothing-3$ & AN2 & 40.7 & & $\mathrm{X}$ & $\mathrm{X}$ & [37] \\
\hline Ris $\varnothing-3$ & AN3 & 42.0 & $\mathrm{X}$ & $\mathrm{X}$ & & [37] \\
\hline Ris $\varnothing-3$ & AN4 & 42.0 & $\mathrm{X}$ & $\mathrm{X}$ & & [37] \\
\hline Ris $\varnothing-3$ & GE7 & 40.9 & & $\mathrm{X}$ & $\mathrm{X}$ & [37] \\
\hline Ris $\varnothing-3$ & II3 & 17.6 & $\mathrm{X}$ & $\mathrm{X}$ & $\mathrm{X}$ & [37] \\
\hline Ris $\varnothing-3$ & II5 & 47.6 & $\mathrm{X}$ & $\mathrm{X}$ & $\mathrm{X}$ & [37] \\
\hline OSIRIS & H09 & 46.1 & & $\bar{X}$ & $\mathrm{X}$ & [37] \\
\hline OSIRIS & $\mathrm{J} 12$ & 26.7 & & $\mathrm{X}$ & $\mathrm{X}$ & [37] \\
\hline REGATE & & 47.0 & & $\mathrm{X}$ & $\mathrm{X}$ & [37] \\
\hline USPWR 16x16 & TSQ002 & 53.2 & & $\mathrm{X}$ & $\mathrm{X}$ & [37] \\
\hline USPWR 16x16 & TSQ022 & 58.1 & & $\mathrm{X}$ & $\mathrm{X}$ & [37] \\
\hline R.E. Ginna & 2 & 51.2 & & $\mathrm{X}$ & $\mathrm{X}$ & [37] \\
\hline R.E. Ginna & 4 & 57.0 & & $\mathrm{X}$ & $\mathrm{X}$ & [37] \\
\hline HBEP & BK363 & 76.0 & & $\mathrm{X}$ & & [33] \\
\hline HBEP & BK365 & 78.3 & & $\mathrm{X}$ & & [33] \\
\hline Tribulation & BN1/3 & 50.7 & & $\mathrm{X}$ & $\mathrm{X}$ & [37] \\
\hline Tribulation & BN1/4 & 50.6 & & $\mathrm{X}$ & $\mathrm{X}$ & [37] \\
\hline Tribulation & $\mathrm{BN} 3 / 15$ & 51.1 & & $\mathrm{X}$ & $\mathrm{X}$ & [37] \\
\hline
\end{tabular}

\section{Validation Results}

\subsection{Validation Standards}

To ensure consistency in the validation process, the standard set of material and behavioral models described above were used in analyzing the LWR integral experiments. Thus the only input data unique to each validation case includes the rod geometry, power history and axial power profile, fast neutron flux and coolant boundary conditions. Generally, before usage as BISON input, experimental data histories were condensed using the Fuel Rod Analysis ToolBox program [40]. A comprehensive list of the standard models and assumptions used, and a more detailed description of each validation case, is available in the BISON validation report [41].
Accurate fuel temperature predictions are essential for fuel performance and safety analysis as all key phenomena taking place in a fuel rod are dominated by the local temperature [42]. Numerous experiments have been conducted with in-situ measurements of the fuel centerline temperature using either a centerline thermocouple or extensometer. The set of experiments considered here are identified in Table 2.

\subsubsection{Beginning of Life Thermal Behavior}

Temperature comparisons during the first rise to power (referred to as Beginning of Life (BOL) in this paper) are significant as they isolate several important aspects of fuel rod behavior before complexities associated with higher burnups are encountered. Accurate prediction of BOL fuel centerline temperature requires accurate models for the fuel and cladding thermal conductivity and gap conductance. The latter depends principally upon the gap gas conductivity and gap width, which in turn depends on models for fuel and cladding thermal expansion and fuel relocation.

Figure 5 summarizes BOL fuel centerline temperature comparisons for the set of experiments in Table 2 where such data are available. Plotted is the predicted versus measured fuel centerline temperature as the rod power is increased during powerup. For all cases considered to date, deviations between BISON predictions and experimental data are less than $\pm 10 \%$. Although measurement uncertainty is rarely reported, reference [35], which is the data report for the Halden IFA-432 experiment, indicates temperature measurement uncertainty of $\pm 1 \%$ over the temperature range of interest. However, the same report indicates that the uncertainty in assembly power is on the order of $\pm 6 \%$. Uncertainties in power and in fuel thermal conductivity are expected to be responsible for most of the uncertainty in the calculated fuel temperature [43].

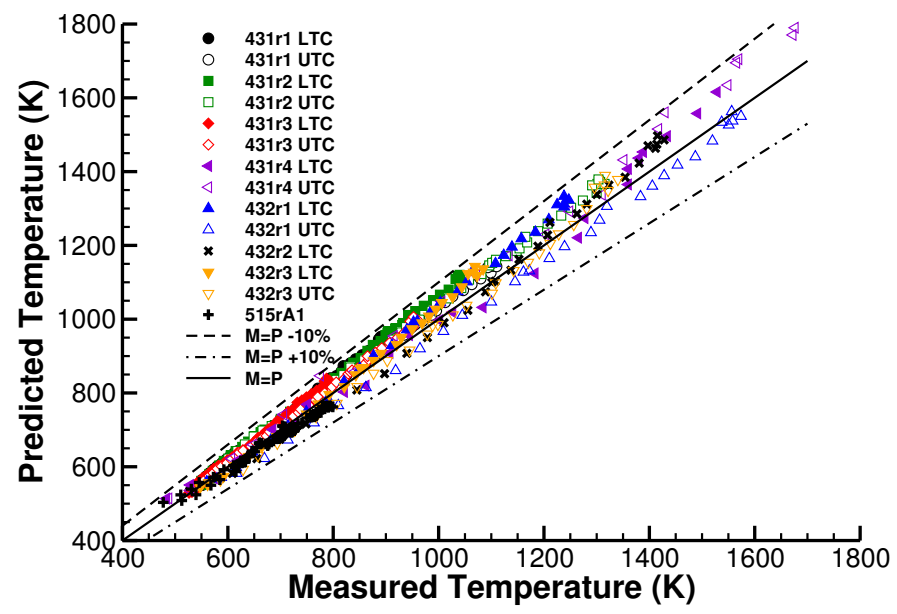

Figure 5: BOL measured vs. predicted fuel centerline temperature for fuel rods in IFA-431, IFA-432, and IFA-515.10. LTC and UTC stand for lower and upper thermocouple measurements, respectively. $\mathrm{M}=\mathrm{P}$ stands for Measured = Predicted.

With one exception, all of the comparisons in Fig. 5 involved experiments and models which were assumed to be axisymmetric. The exception is IFA-431 rod 4, which involved 
3D behavior by design. This comparison is of interest and considered in greater detail here as it provides an opportunity to exercise and validate the 3D analysis capabilities in BISON.

IFA-431 rod 4 was designed to analyze the effects of fuel pellet eccentricity, defined here as the distance a fuel pellet axis of symmetry is radially offset from the cladding axis of symmetry. The fuel rod was $0.62 \mathrm{~m}$ in length with the test pellets of interest located in the upper and lower sections of the rod. The upper test section consisted of 4 annular pellets that were constrained to remain concentric with the axis of symmetry by a molybdenum rod through the fuel centerline. The concentric pellets had an initial fuel-cladding radial gap of $115 \mu \mathrm{m}$. Similarly, the lower test section consisted of 4 pellets that were held eccentrically using another molybdenum rod. The eccentricity value for the case considered here was $90 \mu \mathrm{m}$ resulting in a minimum and maximum initial radial gap of 25 and $205 \mu \mathrm{m}$, respectively. The test pellets (top and bottom) were constrained from radial movement by extending the molybdenum rods into larger diameter pellets above and below the test pellets. These larger pellets were designed to have a very small initial fuel/cladding radial gap $(25 \mu \mathrm{m})$ and thus thermally expand into contact with the cladding, locking the test pellets in place during the initial rise in fuel temperature. Due to the non-axisymmetric geometry of this experiment, it was simulated using two separate 3D models, one for the top section of the rod containing the concentric pellets, and one for the bottom section containing the eccentric pellets [35]. The nearly linear rise to power in the experiment was approximated in the model using a simple linear ramp from $0-20 \mathrm{~kW} / \mathrm{m}$ over a $90,000 \mathrm{~s}$ time period. Since temperature comparisons are made as a function of power, this simplifying approximation is very reasonable.

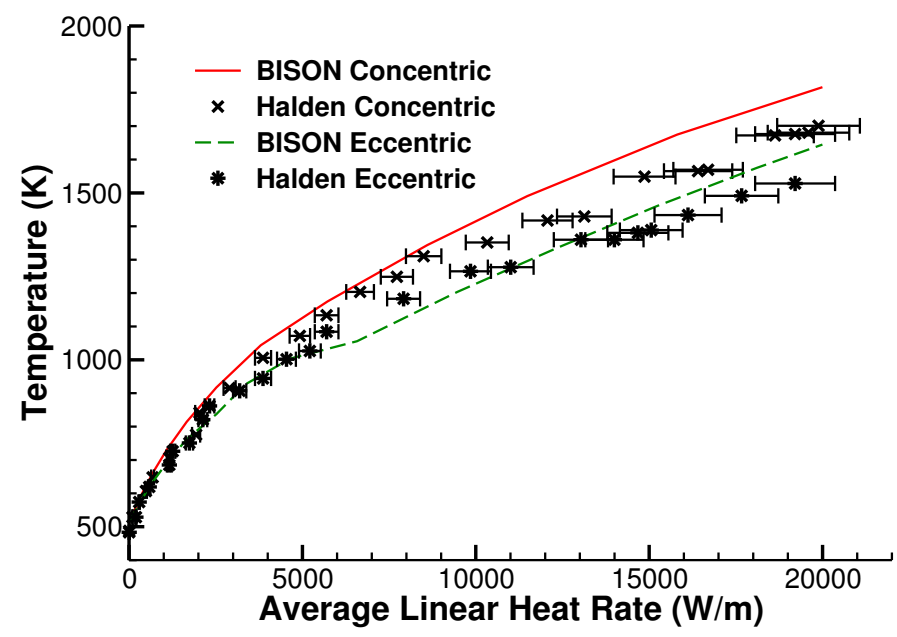

Figure 6: BISON result comparisons to experimental data for the concentric and eccentric test pellets with a $\pm 6 \%$ error bar in linear heat rate.

Figure 6 compares the predicted and measured fuel centerline temperature as a function of rod power, for both the concentric and eccentric pellet configurations. Both experiment and computation indicate that the fuel centerline temperature is lower in the eccentric pellets than in the concentric pellets. For the eccentric pellets, BISON slightly underpredicts the temperature at low power and overpredicts the temperature at higher power. Further note that for the eccentric pellets, the slope of the predicted temperature curve drops at a power of approximately $5 \mathrm{~kW} / \mathrm{m}$. This corresponds to fuel-clad gap closure which, for the eccentric pellets, occurs early in the power rise due to a small initial gap width. For the concentric pellets, temperature predictions are very good at low power but are increasingly overpredicted at higher power. Still, as demonstrated in Fig. 5, predictions are always within $10 \%$ of measurements. Note that efforts are underway to refine the model and potentially improve comparisons, specifically by including the thermal behavior of the molybdenum rods and adding discrete cracking in the fuel pellets.

Figure 7 shows a side-by-side comparison of the computed temperature fields at peak power for the concentric and eccentric models. Also plotted are temperature profiles across the pellet diameter at the axial mid-plane. The difference in the centerline temperature between the concentric and eccentric pellets is because not only are the concentric test pellets higher in temperature (as indicated in Fig. 6) but also the peak temperature of the eccentric pellets is offset from the centerline, where the thermocouple is located. The peak fuel temperature of the concentric pellets occurs, as expected, at the fuel center (TC location) and is approximately $1817 \mathrm{~K}$. The peak temperature for the eccentric pellets is offset $1.4 \mathrm{~mm}$ to the left of the TC location (see Fig. 7) and is approximately $1644 \mathrm{~K}$ [36].

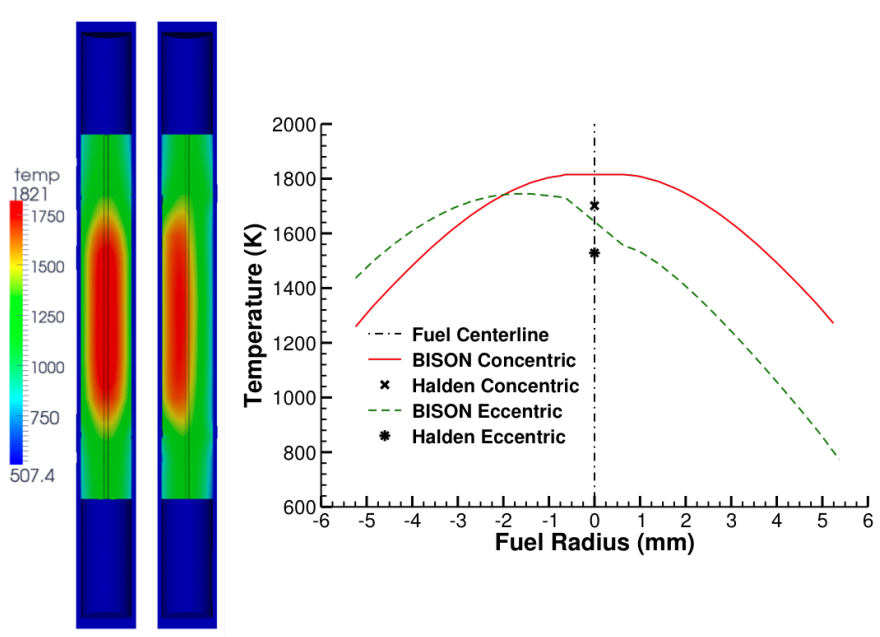

Figure 7: Temperature contour plots of the concentric (left) and eccentric (right) pellets and the fuel temperature profile across the diameter of the pellets at the fuel axial mid-plane.

\subsubsection{Through-Life Fuel Thermal Behavior}

Four cases in Table 2 contained through-life temperature data: IFA-515.10 Rod A1 and IFA-562 Rods 15, 16 and 17 [38, 39]. The four cases were composed of annular $\mathrm{UO}_{2}$ fuel pellets enclosed in Zircaloy- 2 cladding. The through-life temperature measurements were obtained using centerline expansion thermometers (ET) that were placed inside the inner diameter of the fuel stack. These ETs spanned the entire length of the fuel stack and therefore provided average fuel centerline measurements as derived from their elongation. These rods were irradiated to 


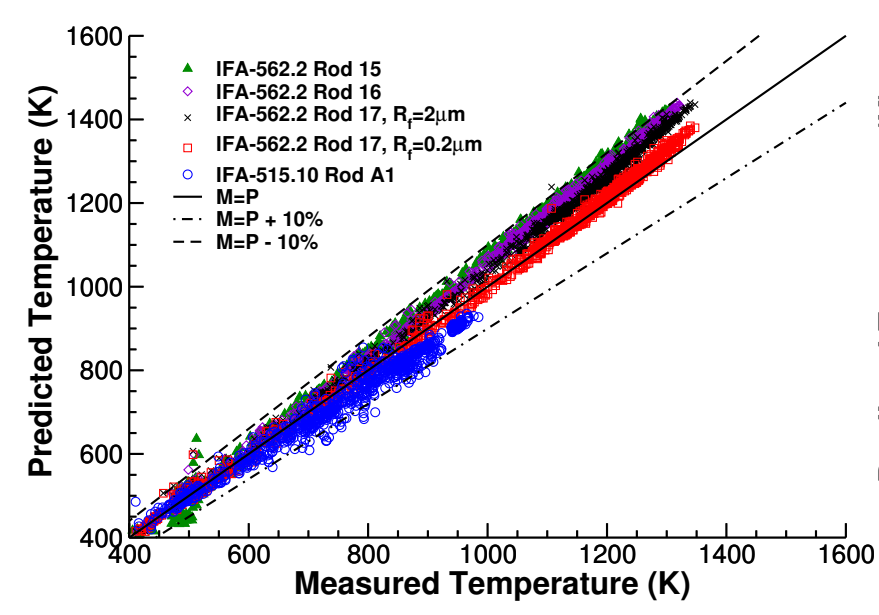

(a)

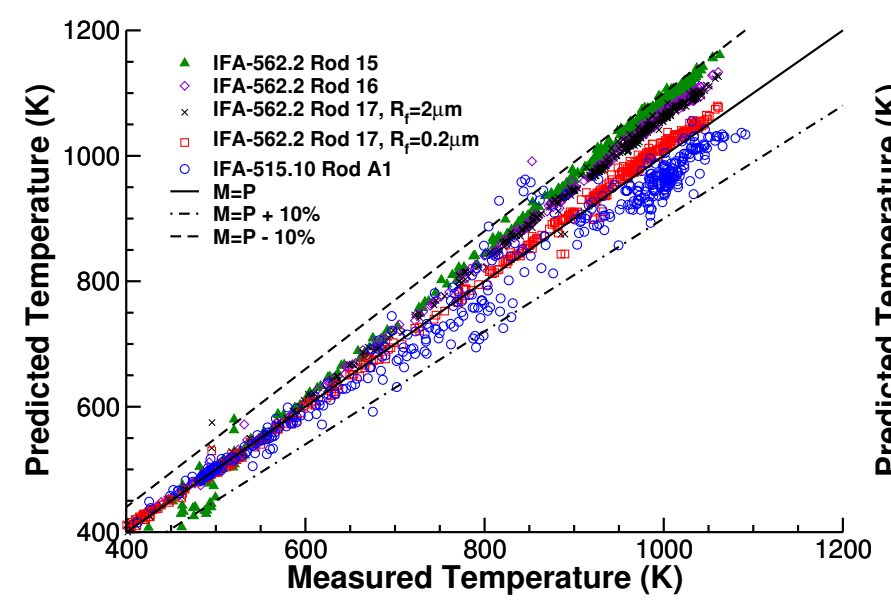

(c)

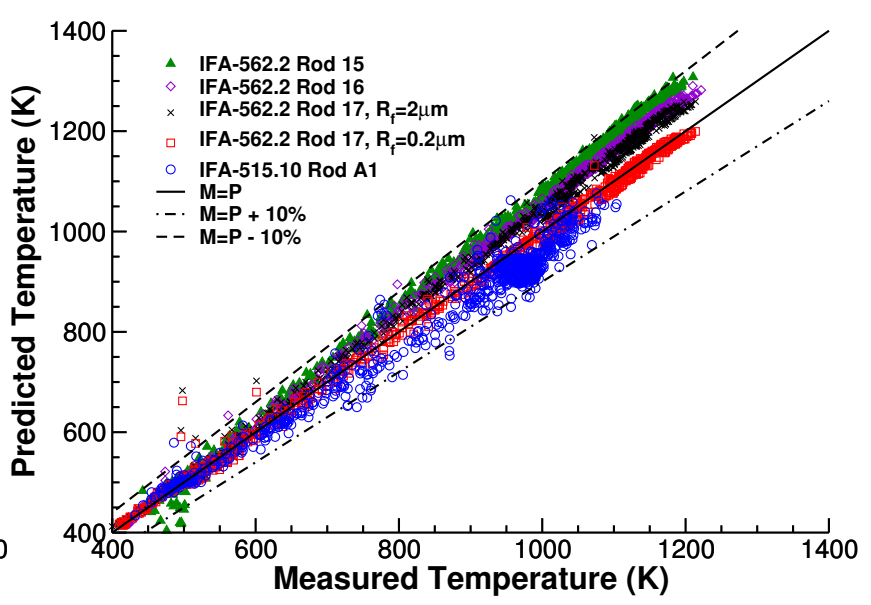

(b)

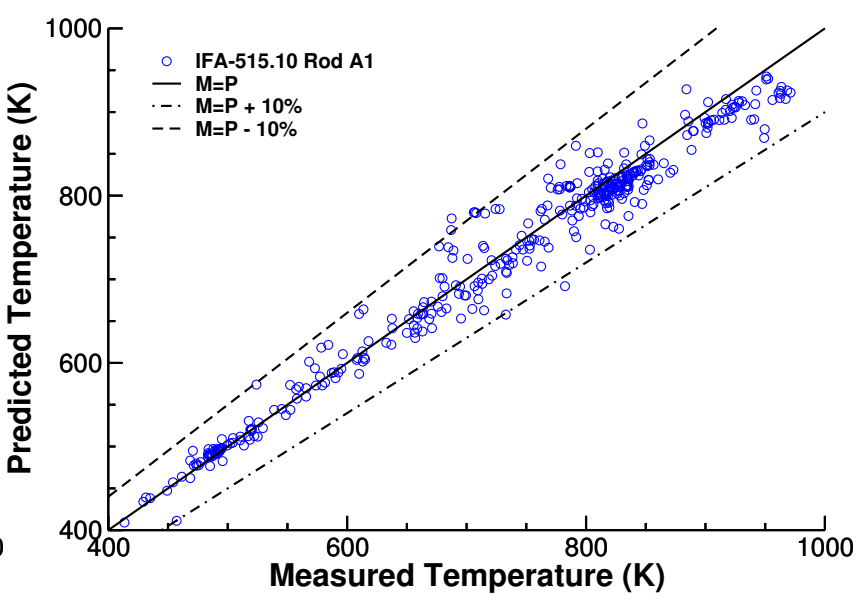

(d)

Figure 8: Comparison of the measured vs. predicted fuel centerline temperature for through-life rods for four different burnup ranges: (a) $0 \leq$ Bu $<20$, (b) $20 \leq$ $\mathrm{Bu}<40$, (c) $40 \leq \mathrm{Bu}<60$, and (d) $\mathrm{Bu} \geq 60 \mathrm{MWd} / \mathrm{kgUO}_{2}$. The $\mathrm{R}_{f}$ parameter in the IFA-562.2 Rod 17 labels indicate the fuel roughness used in the simulation. $\mathrm{M}=\mathrm{P}$ stands for Measured $=$ Predicted.

high burnups, 75.5 MWd//gUO 2 for IFA-515.10 Rod A1, and an average of $49.4 \mathrm{MWd} / \mathrm{kgUO}_{2}$ for the IFA-562.2 rods. To illustrate results, measured vs. predicted plots are given for four different burnup increments: $0 \leq \mathrm{Bu}<20,20 \leq \mathrm{Bu}<40,40 \leq$ $\mathrm{Bu}<60$, and $\mathrm{Bu} \geq 60 \mathrm{MWd} / \mathrm{kgUO}_{2}$ as shown in Figure 8 .

For the IFA-515.10 Rod A1 irradiation, it is observed that the bulk of the data points for all burnup ranges are within the $\pm 10 \%$ error bands as illustrated by the blue circles. At low temperatures the deviation between BISON and the experiment is small with the majority of points falling close to the measured equals predicted $(\mathrm{M}=\mathrm{P})$ line. As irradiation progresses it is observed that the majority of the calculated temperatures remain within $10 \%$ of the experimental values. This indicates that BISON's fuel thermal conductivity model accurately captures the degradation of the thermal conductivity as a function of burnup. The centerline temperatures are strongly influenced by the fuel-to-clad gap model. As mentioned previously, BISON utilizes a model that is comprised of three separate components: gas conductance, solid-to-solid conductance, and radiative heat transfer. Prior to contact, the gas conductance term dominates and is influenced by the gap width, roughness of the fuel and clad, thermal conductivity of the gas mixture (including fission gases), and the jump distances. After contact occurs the solid-to-solid component further influences the calculated centerline temperatures and is a function of the surface roughness and thermal conductivity of the fuel and clad materials, the hardness of the softer material and the contact pressure.

The influence of gap heat transfer on centerline temperatures is illustrated by examining the results of the IFA-562.2 rods in Figure 8. The documentation of the IFA-562.2 irradiation did not provide fuel roughness measurements. Therefore, for rods 15,16 , and 17 the BISON default value of $2 \mu \mathrm{m}$ was assumed. As expected, the calculated temperatures for all of the IFA-562.2 rods analyzed are similar since they were subjected to a nearly identical power history. Once again almost all of the predictions fall within $\pm 10 \%$ of the experimental values. The reason for including a second simulation of Rod 17 with a fuel roughness of $0.2 \mu \mathrm{m}$ was to highlight two things: (1) changes in simulation temperatures due to interpretation of the experiment and (2) the strong influence fuel roughness has 
on the gap conductance during irradiation. The value of 0.2 $\mu \mathrm{m}$ was chosen because it was the reported value for the IFA515.10 Rod A1 experiment [38]. By using a surface roughness of $0.2 \mu \mathrm{m}$ it is observed that the calculated temperatures are approximately $5 \%$ below those predicted when using the default surface roughness. This temperature reduction is directly related to the gap conductance. As the fuel roughness increases, the ability to transfer heat across the gap is decreased, resulting in higher fuel temperatures when the gap is open or closed. As stated above, both the gas and solid-to-solid conductance models are influenced by the fuel and clad roughnesses. Note that a $0.2 \mu \mathrm{m}$ roughness is quite smooth suggesting there might be an error in the reporting of this value. The authors have contacted the experimentalists at the Halden reactor inquiring about the validity of the fuel roughness reported in [38] for experiment IFA-515.10 Rod A1.

To illustrate the complexities of simulating a through-life irradiation, a more in depth description of IFA-515.10 Rod A1 is provided. The IFA-515.10 Rod A1 experiment was irradiated in the Halden Boiling Water Reactor (HBWR) for a little over 6 years to a discharge burnup of approximately 75.5 $\mathrm{MWd} / \mathrm{kgUO}_{2}$. Figure 9(a) shows the input power history and Figure 9(b) the average centerline temperature comparison between BISON and the Halden experiment for the duration of irradiation. The complexity of the irradiation history is evident. As shown in Figure 8, BISON predicts average centerline temperature within $\pm 10 \%$ of the measured values. When the reactor is at zero power, some measured temperatures are clearly nonphysical and were not included in the comparison.

Through-life predictions of centerline temperature assess the validity of models used to model gap heat transfer and fuel thermal conductivity evolution with irradiation. It has been shown that the models included in BISON capture this behavior.

\subsubsection{Thermal Ramp Behavior}

Five of the experiments listed in Table 2 (Ris $\varnothing-3$ rods AN3, AN4, II3, II5 and IFA-597.3 rod 8) include measurements of fuel centerline temperature during power ramps following base irradiation. These experiments include rods with burnups ranging from 14.5 to $61.6 \mathrm{MWd} / \mathrm{kgU}$. In all cases, experimental rods were refabricated for installation in the test reactor by shortening mother rods. The purpose of power ramp experiments are to examine rod behavior due to short, high power transients under a variety of initial conditions. The initial conditions for a particular experiment are determined by the base irradiation history and the conditions of refabrication. For simplicity, BISON simulations assume the geometry of the shortened rod for both the base irradiation and ramp test calculations while applying the full base and ramp power histories.

Similar to Figures 5 and 8, Figure 10 compares measured and predicted fuel centerline temperatures for the five ramp test experiments. Comparisons are reasonable; however, some points fall outside the $\pm 10 \%$ error bands. Two modeling assumptions could be a source of uncertainty in the temperature predictions during the ramp: (1) using the shortened rod geometry for the base irradiation and (2) approximations involved in

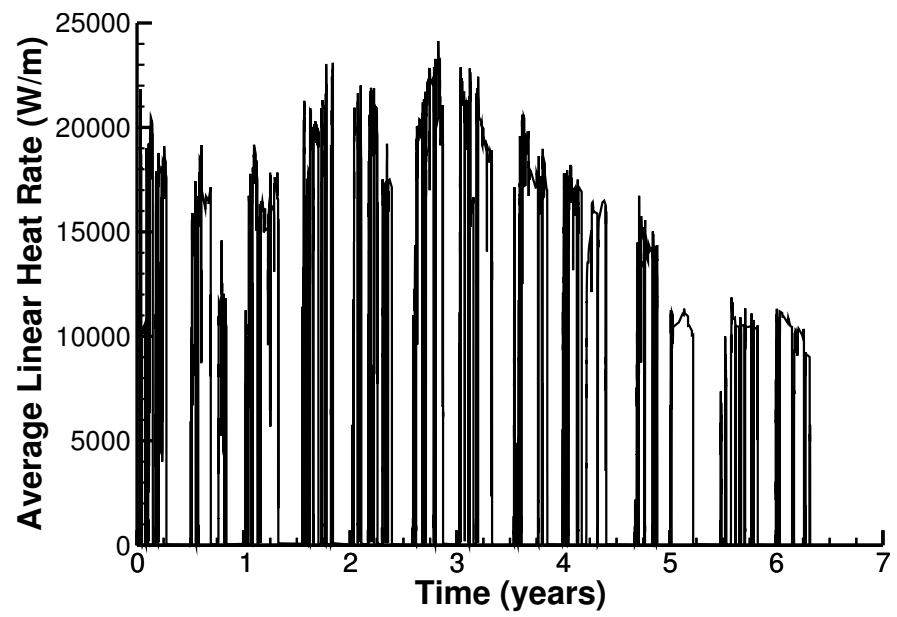

(a)

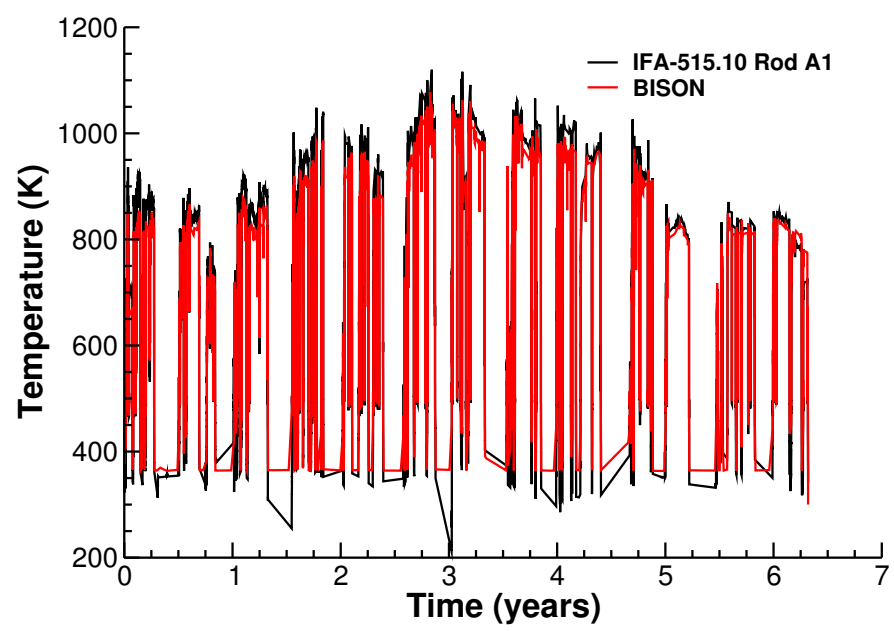

(b)

Figure 9: (a) The average linear heat rate and (b) comparison of predicted and measured fuel centerline temperature histories for the Halden IFA-515.10 irradiation.

simulating the rod refabrication process. Refabrication in BI$\mathrm{SON}$ is accounted for by specifying the refabrication temperature, pressure, and volume to determine the initial gas content of the refabricated rod. Simulating the base irradiation on the geometry of the refabricated rod leads to some discrepancies in the plenum volume at the time of refabrication. This brings about inaccuracies in the calculation of the initial moles of gas in the refabricated rod, consequently in the calculated gas pressure and fuel temperature through the gap conductance. Comparisons of BISON predicted rod internal pressure to available experimental data, which can be found in reference [41], concur with this explanation of discrepancies for the low-temperature comparisons in Figure 10. 


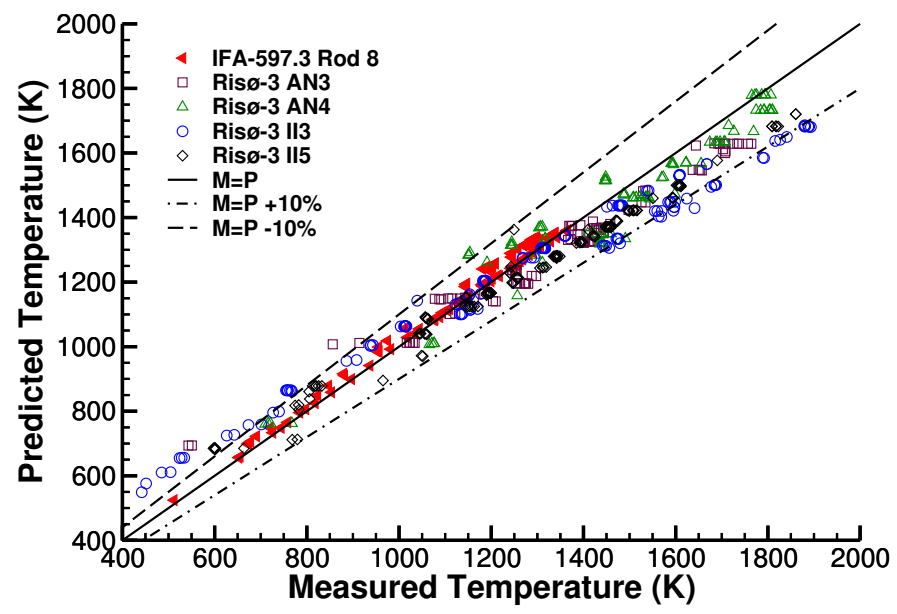

Figure 10: Comparison of the measured vs. predicted fuel centerline temperature for fuel rods that experienced power ramps. $\mathrm{M}=\mathrm{P}$ stands for Measured = Predicted.

To describe a typical power ramp experiment and provide time history comparisons for both fuel centerline temperature and fission gas release (explained below), the Ris $\varnothing-3$ AN3 case is considered in greater detail. This experiment was conducted at the Ris $\varnothing$ DR3 test reactor and utilized a re-fabricated fuel rod that was base irradiated in the Biblis-A pressurized water reactor $[33,44]$. The mother rod, CB8, was irradiated over four reactor cycles to a burnup of $40.9 \mathrm{MWd} / \mathrm{kgU}$. The refabricated rod, CB8-2R, was instrumented with a fuel centerline thermocouple and pressure transducer and then subjected to a 72 hour ramp test with a peak power of approximately $40 \mathrm{~kW} / \mathrm{m}$ and a final burnup of $41.8 \mathrm{MWd} / \mathrm{kgU}$. The average fuel rod linear heat rate during the ramp test is illustrated in Figure 11.

The plot in Figure 12 shows a scaled section of the geometry, materials, computational mesh and a typical temperature contour result for the section of fuel and cladding near the top of the fuel pellet stack. The temperature was measured/calculated at a point approximately $36.4 \mathrm{~mm}$ from the top of the fuel stack, approximately 1.5 pellet lengths above the bottom of the thermocouple hole (see black dot on contour plot).

A comparison of the predicted and measured fuel centerline temperature histories for the Ris $\varnothing-3$ AN3 ramp experiment are also shown in Figure 11. BISON predicts the fuel centerline temperature within $10 \%$ of the measurements throughout the duration of the ramp. Note that BISON systematically underpredicts the fuel centerline temperature throughout the ramp test. This is very likely due to an underprediction of the fission gas release at the end of base irradiation (beginning of ramp) where the measured and predicted release was roughly 7 and $1.5 \%$, respectively. Increased fission gas results in degradation of the gap conductance, leading to higher fuel temperatures.

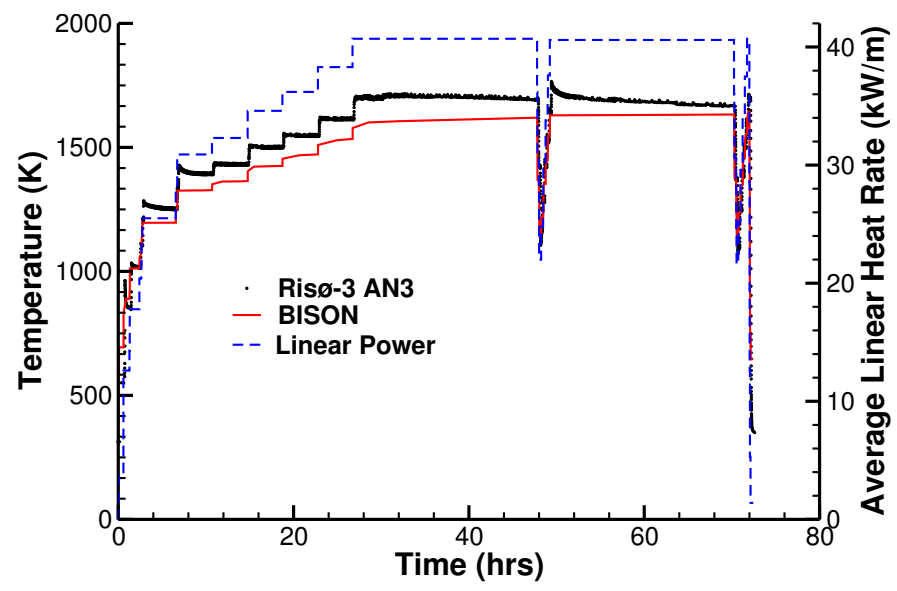

Figure 11: BISON fuel centerline temperature compared to Ris $\emptyset-3$ AN3 experimental data. The thermocouple location is indicated on the inset temperature contour plot. The power history for the calculation is also shown.

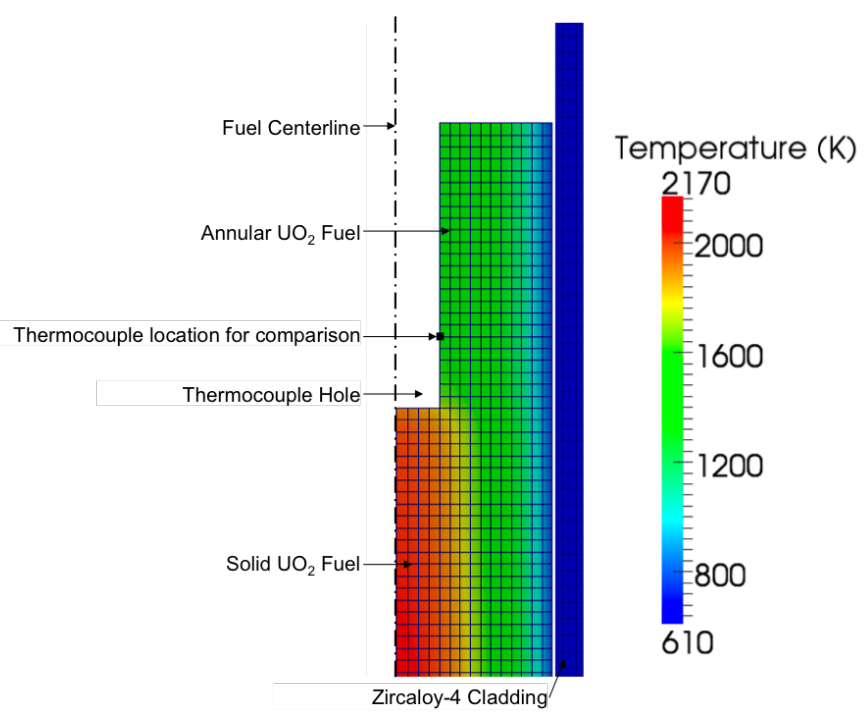

Figure 12: A section of the Ris $\varnothing-3$ AN3 rod showing the geometry, materials and computational mesh. Temperature results are also given at approximately 40 hours into ramp test. Note that the radial dimension is scaled by a factor of 5 .

\subsection{Fission Gas Release}

BISON predictions of integral fuel rod FGR from simulations of the validation cases listed in Table 2 have been compared to the available experimental data. Due to the inherent uncertainties of fission gas behavior modeling, a deviation of fuel performance code predictions from the experimental data within a factor of about 2 up and down is generally regarded as satisfactory [33, 34, 45]. A recent uncertainty evaluation study supported the estimation of a factor of 2 as the range of tolerance for high FGR values, yet pointing out that higher deviations may be expected for calculated FGR around $10 \%$ and lower [46]. The comparison of BISON calculations and experimental data of integral FGR at the fuel rod end-of-life (EOL) is summarized in Figure 13 with the data tabulated in Table 3. BISON's accuracy in predicting FGR appears to be generally consistent with state-of-the-art modeling and with the inherent 
uncertainties [33, 34, 45, 46]. A stronger discrepancy between calculated and experimental FGR is observed for Ris $\varnothing-3$ II5. This is associated with an underprediction of temperature, and will be the subject of further investigation.

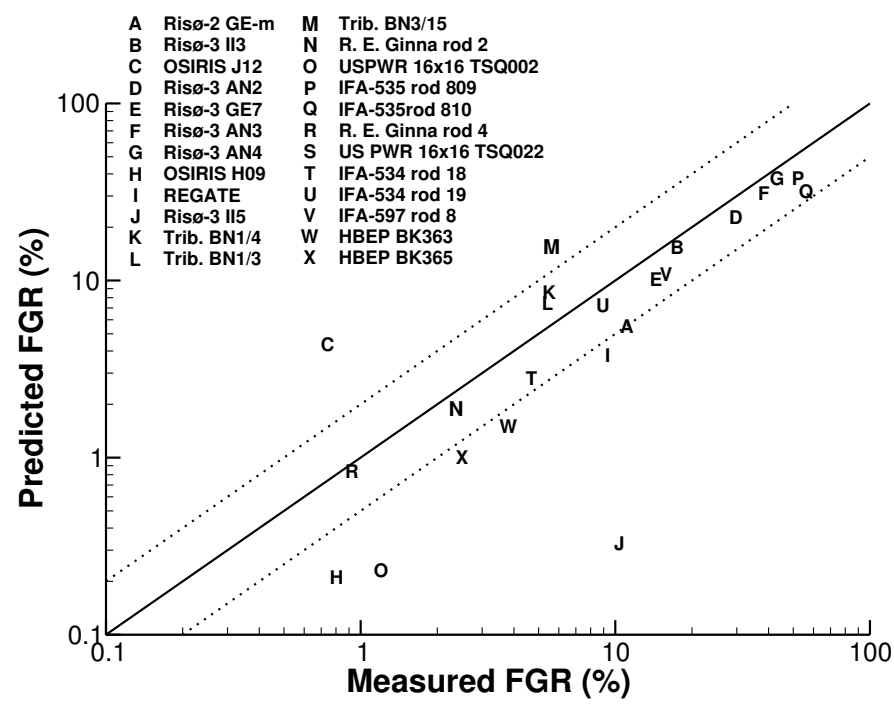

Figure 13: Fission gas release measured versus predicted comparisons with a factor of 2 error band. Note that case S, identified in the legend, does not appear within the domain of the plot since the measured value was $0.9 \%$ while the computed value was zero.

Table 3: Fission gas release measured versus predicted comparisons.

\begin{tabular}{|c|c|c|c|c|}
\hline Experiment & Rod & $\begin{array}{c}\text { Final } \\
\text { Burnup } \\
(\mathrm{MWd} / \mathrm{kgU})\end{array}$ & $\begin{array}{l}\text { Measured } \\
\text { FGR } \\
(\%)\end{array}$ & $\begin{array}{l}\text { Predicted } \\
\text { FGR } \\
(\%)\end{array}$ \\
\hline $\operatorname{Ris} \emptyset-2$ & GE-m & 15.8 & 11.1 & 5.5 \\
\hline Ris $\varnothing-3$ & II3 & 17.6 & 17.4 & 15.5 \\
\hline OSIRIS & $\mathrm{J} 12$ & 26.7 & 0.7 & 4.4 \\
\hline $\operatorname{Ris} \varnothing-3$ & AN2 & 40.7 & 29.7 & 22.8 \\
\hline Ris $\varnothing-3$ & GE7 & 40.9 & 14.4 & 10.2 \\
\hline Ris $\varnothing-3$ & AN3 & 42.0 & 38.3 & 31.3 \\
\hline $\operatorname{Ris} \emptyset-3$ & AN4 & 42.0 & 43.0 & 50.5 \\
\hline OSIRIS & H09 & 46.1 & 0.8 & 0.2 \\
\hline REGATE & & 47.0 & 9.3 & 3.8 \\
\hline Ris $\emptyset-3$ & II5 & 47.6 & 10.3 & 0.3 \\
\hline Tribulation & BN1/4 & 50.6 & 5.5 & 8.6 \\
\hline Tribulation & BN1/3 & 50.7 & 5.4 & 7.5 \\
\hline Tribulation & $\mathrm{BN} 3 / 15$ & 51.1 & 5.6 & 15.6 \\
\hline R.E. Ginna & 2 & 51.2 & 2.4 & 1.9 \\
\hline USPWR 16x16 & TSQ002 & 53.2 & 1.2 & 0.2 \\
\hline IFA-535 & 809 & 54.4 & 52.0 & 38.0 \\
\hline IFA-535 & 810 & 54.4 & 56.0 & 32.0 \\
\hline R.E. Ginna & 4 & 57.0 & 0.9 & 0.8 \\
\hline USPWR 16x16 & TSQ022 & 58.1 & 0.9 & 0.0 \\
\hline IFA-534 & 18 & 59.0 & 4.7 & 2.8 \\
\hline IFA-534 & 19 & 59.0 & 8.9 & 7.2 \\
\hline IFA-597.3 & 8 & 68.1 & 15.8 & 4.0 \\
\hline HBEP & BK363 & 76.0 & 3.8 & 1.5 \\
\hline HBEP & BK365 & 78.3 & 2.5 & 1.0 \\
\hline
\end{tabular}

The comparison of integral fuel rod FGR as a function of time for the Risø-3 AN3 ramp test is shown in Figure 14. The accuracy of the FGR representation obtained with BISON for this case is the same or better than the state of the art [33]. The rapid increases (bursts) of FGR are qualitatively reproduced, pointing out a consistent representation of the release kinetics, albeit their magnitute is under-predicted. Indeed, it may be postulated that the magnitude of the release bursts measured via pressure transducer is over-estimated. A rapid increase of the measured fuel rod inner pressure may be partly associated with gap and cracks opening during a power drop, and consequent delayed detection of gas released from the fuel prior to the transient $[33,47,48]$.

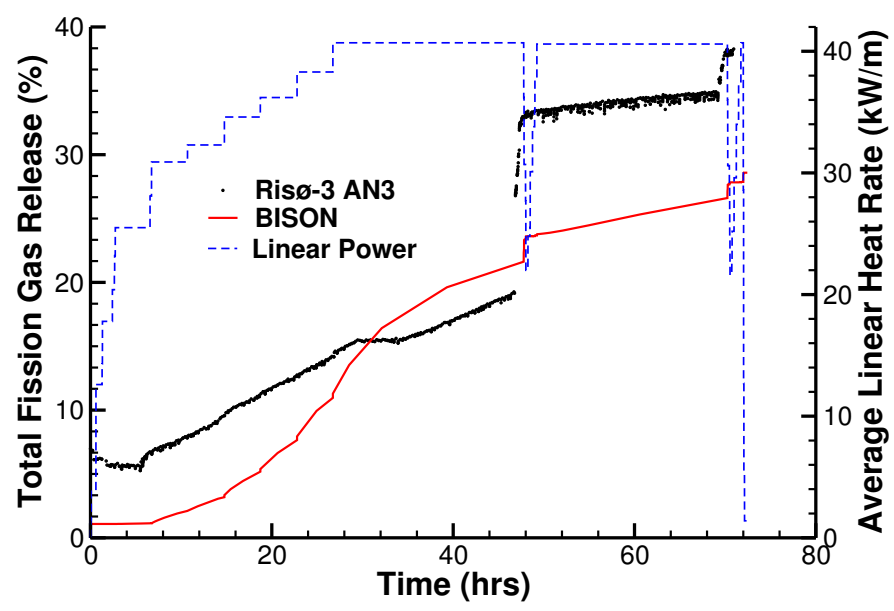

Figure 14: BISON total fission gas released compared to Ris $\emptyset-3$ AN3 experimental data obtained via pressure transducer.

\subsection{Rod Diameter}

Accurate simulation of the mechanical behavior of fuel rods is important when attempting to make predictions about cladding structural integrity, for example as a result of pellet cladding mechanical interaction (PCMI). Eleven of the experiments listed in Table 2 included rod diameter measurements. All measurements were made post irradiation in a hot cell, with a few including measurements following both base irradiation and after a power ramp.

BISON simulations were performed for comparison to each of these experiments. In all cases, the fuel pellet stack was simulated as a single smeared fuel column. This was done principally for simplicity in this initial validation study, since BISON is capable of discrete pellet analysis.

Table 4 summarizes the rod diameter comparisons following base irradiation, in terms of the difference between the measured and predicted values. As indicated in the table, comparisons are made either at the rod axial midplane or averaged over the rod length, based on the available experimental data. The comparisons are separated by cladding type (Zircaloy-4 and Zircaloy-2) and ordered according to the final burnup. The diameter comparisons are also plotted in Figure 15 in terms of diameter change vs burnup. In general, the results indicate a tendency to overpredict the diameter reduction which occurs early in life and more significantly overpredict the diameter increase which occurs late in life.

The early life comparisons typically occur before fuel-clad contact, when clad deformation is dominated by clad creep- 
down; comparisons thus indicate a tendency to overpredict clad creep rates. Although the number of low burnup points are limited, diameter comparisons indicate this overprediction is more severe for Zircaloy-2 than Zircaloy-4. Reasons for this overprediction could include large variations and uncertainty which exist in clad microstructure and the lack of an anisotropic creep model in BISON. Comparison to separate effects clad creep experiments, such as the Halden IFA-585 test [49], are underway to better understand and improve predictions.

At higher burnups, following fuel-clad contact, clad diameter increase is controlled by the mechanical behavior of the fuel. The fact that the clad diameter change (measured - predicted) is significantly negative indicates a strong tendency to overpredict fuel radial growth. BISON's over prediction of final rod diameter may be partially due to its lack of a relocation recovery model, which would recover a portion of the relocation strain upon fuel-cladding contact. Uncertainty in the initial fuel porosity and densification during irradiation provide further explanation for poor diameter comparisons. Fuel creep, which has been neglected in the present paper, is also expected to play a significant role.

To explore some of these issues a simple parametric study was performed for a single validation case (R. E. Ginna Rod 2) with three separate modifications to the base calculation: 1) fuel relocation was deactivated, 2) fuel relocation was active but relocation strains were reduced by $50 \%$ and, 3) fuel relocation was active but fuel densification was increased by $0.5 \%$. The partial reduction in relocation strain was motivated by the relocation recovery model in the FRAPCON fuel performance code, which recovers (by default) $50 \%$ of the relocation strain upon contact [50]. Referring to the diameter change (measured - predicted) parameter in Table 4, which was $-71.8 \mu \mathrm{m}$ for the Ginna Rod 2 case, simply removing relocation strain from the calculation dropped that difference to $-19.6 \mu \mathrm{m}$, demonstrating that the BISON relocation model, which is simplistic and highly empirical, has a substantial effect on the rod diameter comparison. Recovering half of the relocation strain resulted in a final clad diameter change of $-45 \mu \mathrm{m}$, demonstrating the importance of including relocation recovery behavior. Simply increasing the fuel densification by $0.5 \%$ resulted in a final diameter change of $-60.1 \mu \mathrm{m}$, indicating that uncertainties in this parameter will have a smaller, but still significant effect.

Ongoing PCMI validation efforts will focus on the BISON fuel creep, relocation and densification models. It is worth noting that prediction of cladding diametral strain is in general a difficult area for fuel performance codes, as demonstrated by international benchmark exercises [33, 34].
Table 4: Rod diameter comparisons following base irradiation

\begin{tabular}{|c|c|c|c|c|}
\hline Case Description & $\begin{array}{l}\text { Clad } \\
\text { Type }\end{array}$ & $\begin{array}{c}\text { Axial } \\
\text { Comparison } \\
\text { Location }\end{array}$ & $\begin{array}{l}\text { Rod Average } \\
\text { Burnup } \\
(\mathrm{MWd} / \mathrm{kgU})\end{array}$ & $\begin{array}{c}\text { Diameter Change } \\
\text { (Meas. - Pred.) } \\
(\mu \mathrm{m})\end{array}$ \\
\hline Tribulation BN1/4 & Zry-4 & Midplane & 19.7 & 16.4 \\
\hline Tribulation BN1/3 & Zry-4 & Midplane & 20.2 & 7.1 \\
\hline OSIRIS J12 & Zry-4 & Average & 23.9 & -18.1 \\
\hline Tribulation BN3/15 & Zry-4 & Midplane & 37.7 & -71.1 \\
\hline Ris $\emptyset-3$ AN2 & Zry-4 & Average & 40.6 & -16.6 \\
\hline OSIRIS H09 & Zry-4 & Average & 46.1 & -42.9 \\
\hline REGATE & Zry-4 & Average & 47.0 & 6.5 \\
\hline Tribulation BN1/4 & Zry-4 & Midplane & 50.6 & -53.2 \\
\hline Tribulation BN1/3 & Zry-4 & Midplane & 50.7 & -70.8 \\
\hline Tribulation BN3/15 & Zry-4 & Midplane & 51.1 & -84.8 \\
\hline R. E. Ginna Rod 2 & Zry-4 & Average & 51.2 & -71.8 \\
\hline USPWR TSQ002 & Zry-4 & Average & 53.2 & -26.3 \\
\hline R. E. Ginna Rod 4 & Zry-4 & Average & 57.0 & -69.2 \\
\hline USPWR TSQ022 & Zry-4 & Average & 58.1 & -40.4 \\
\hline Ris $\emptyset-2$ GE-m & Zry-2 & Average & 15.5 & 35.8 \\
\hline Ris $\emptyset-3$ II3 & Zry-2 & Average & 16.4 & 32.8 \\
\hline Ris $\varnothing-3$ II5 & Zry-2 & Midplane & 39.0 & -78.8 \\
\hline Ris $\emptyset-3$ GE7 & Zry-2 & Average & 40.9 & 34.1 \\
\hline
\end{tabular}

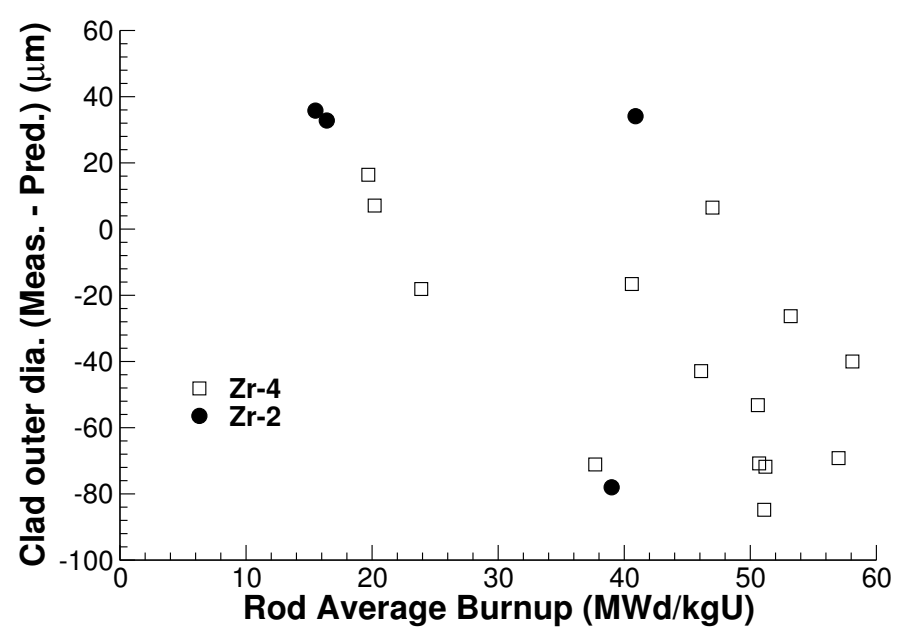

Figure 15: The difference between measured and predicted rod diameter as a function of burnup during base irradiation.

Eight of the cases from Table 4 included power ramp testing following base irradiation, with post-ramp diameter comparisons (measured - predicted) summarized in Table 5. To isolate comparison to only the ramp portion of the experiment, the diameter differences at the end of the base irradiation (Table 4) were subtracted from those at the end of the ramp (Table 5) and plotted in Figure 16 as a function of base irradiation burnup. Comparisons are very reasonable for low burnup fuel but grow steadily worse at higher burnups. Note that, in contrast to the behavior during base irradiation, where the radial fuel growth is overpredicted, for power ramps the measured value generally exceeds the prediction indicating the fuel diameter change is underpredicted. During relatively short duration power ramps, the radial growth of the fuel is driven mainly by thermal expansion and gaseous swelling. Indeed, recent sensitivity analysis indicated that uncertainties inherent in gaseous swelling modeling have a significant impact on attainable accuracy of clad diameter predictions during power ramps [46]. In particu- 
lar, a sensitivity study was presented in [46], wherein the effect of modeling uncertainties associated with fission gas swelling (and coupled FGR) on cladding diameter calculations for the Ris $\varnothing-3$ GE7 experiment was estimated. The study pointed out a variability of about $150 \mu \mathrm{m}$ on predicted peak cladding displacement at the end of irradiation corresponding to such uncertainties. Although limited to one fuel rod experiment, the study supports the hypothesis of a dominant contribution of gaseous swelling modeling uncertainties to the discrepancies of current cladding diameter predictions. Further investigation and sensitivity analysis is necessary in order to confirm such hypothesis.

Table 5: Rod diameter comparisons following power ramps

\begin{tabular}{|c|c|c|c|c|}
\hline Case Description & $\begin{array}{l}\text { Clad } \\
\text { Type }\end{array}$ & $\begin{array}{c}\text { Axial } \\
\text { Comparison } \\
\text { Location }\end{array}$ & $\begin{array}{l}\text { Rod Average } \\
\text { Burnup } \\
(\mathrm{MWd} / \mathrm{kgU})\end{array}$ & $\begin{array}{c}\text { Diameter Change } \\
\text { (Meas. - Pred.) } \\
(\mu \mathrm{m})\end{array}$ \\
\hline Tribulation BN1/3 & Zry-4 & Midplane & 20.4 & 12.3 \\
\hline OSIRIS J12 & Zry-4 & Peak & 26.7 & -1.4 \\
\hline Ris $\emptyset-3$ AN2 & Zry-4 & Peak & 40.7 & 24.7 \\
\hline REGATE & Zry-4 & Peak & 47.0 & 88.4 \\
\hline Ris $\emptyset-2$ GE-m & Zry-2 & Average & 15.8 & 50.6 \\
\hline Ris $\varnothing-3$ II3 & Zry-2 & Average & 17.6 & 31.7 \\
\hline Ris $\varnothing-3$ GE7 & Zry-2 & Peak & 40.9 & 58.9 \\
\hline Ris $\varnothing-3$ II5 & Zry-2 & Midplane & 47.6 & -26.7 \\
\hline
\end{tabular}

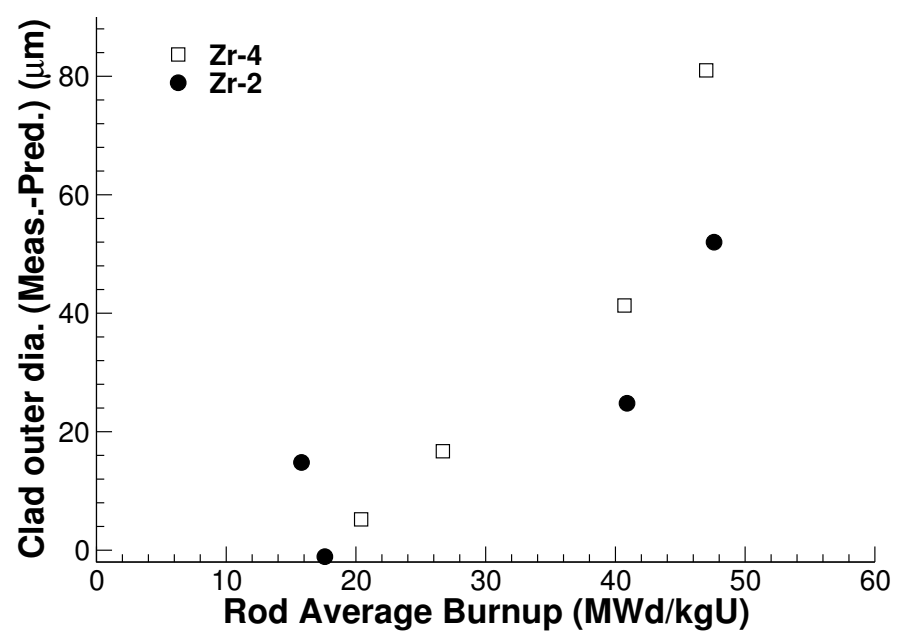

Figure 16: The difference between measured and predicted rod diameter during ramp testing, as a function of base irradiation burnup. To isolate comparisons to diameter changes occurring during the ramp only, diameter differences at the end of base irradiation were subtracted from those at the end of the ramp.

Ris $\varnothing-3$ GE7, considered in more detail here, is a typical example of an experiment where rod diameter is measured both at the end of base irradiation and following a power ramp. This experiment involved a rod segment that underwent four cycles of base irradiation to a burnup of $39.6 \mathrm{MWd} / \mathrm{kgU}$ in the Quad Cities-1 BWR, followed by a power ramp in the Ris $\varnothing$ DR3 test reactor. The cladding was stress relieved Zircaloy-2 with a bonded zirconium liner. The liner was assumed to have the properties of Zircaloy-2 in the simulation. The fuel segment was neither refabricated nor punctured prior to irradiation in the test reactor.

The power history since the beginning of the power ramp, shown in Figure 17, includes a 6-hour conditioning period at approximately $23 \mathrm{~kW} / \mathrm{m}$, a 15 minute power ramp, and then a 4-hour hold at $35.5 \mathrm{~kW} / \mathrm{m}$. Figure 18 shows the axial power profile during both the base irradiation and power ramp. In contrast to the base-irradiation profile, which is relatively flat, there is a large axial gradient in the ramp profile.

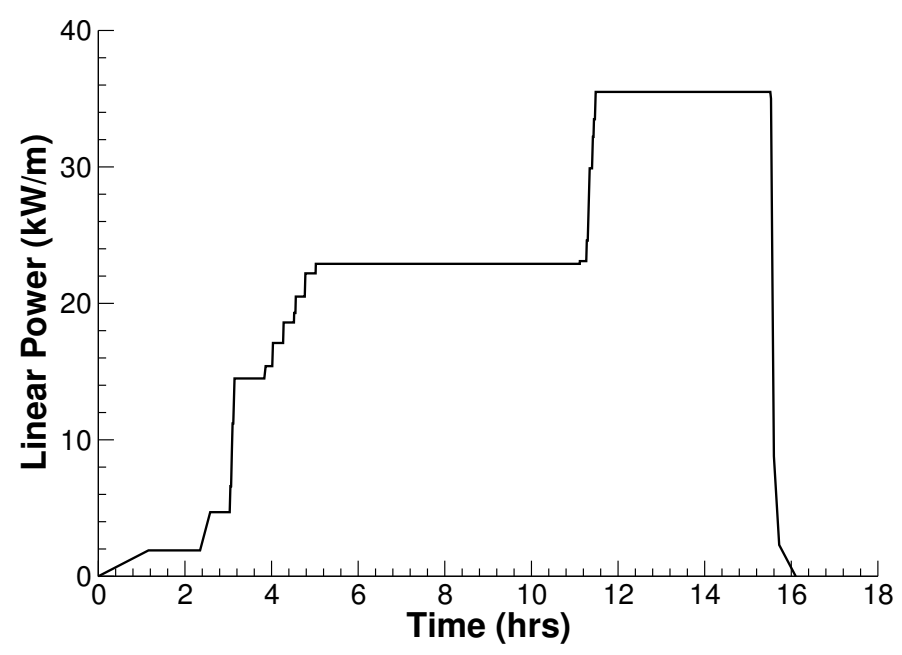

Figure 17: Risø-3 GE7 power history during power ramp.

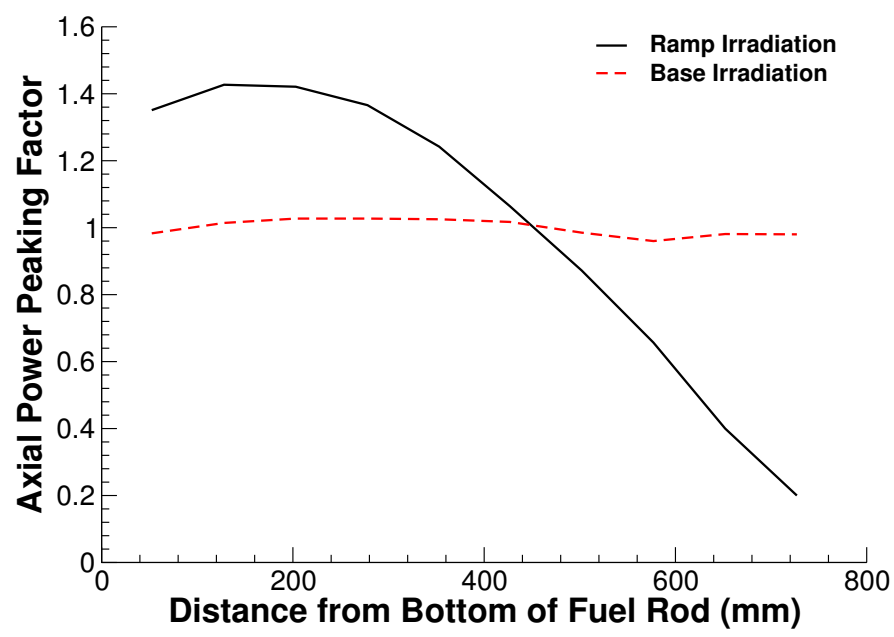

Figure 18: Ris $\emptyset-3$ GE7 axial power profile during base irradiation and power ramp.

Figure 19 compares the predicted and measured rod diameter as a function of rod length, both following the base irradiation and power ramp. The diameter of the preirradiated rod is shown for reference. BISON appears to overpredict clad creep rates during base irradiation, resulting in a decrease in rod diameter roughly double the measured value. Note that the predicted axial diameter profile has a shape very similar to the axial power profile, indicating the fuel has been in contact with the clad during base irradiation. This is indeed the case as gap closure is predicted to occur at a burnup of approximately 28 $\mathrm{MWd} / \mathrm{kgU}$. On the other hand, the measured diameter profile following base irradiation shows no evidence of contact; this is not surprising based on much lower measured clad creep down. 
Comparing the measured and predicted diameter change during the ramp test indicates BISON does a reasonable job of predicting the transient mechanical behavior. Permanent change in the clad diameter results from both creep and instantaneous plasticity and is driven principally by thermal expansion and gaseous swelling of the fuel. As noted above, fission gas re-

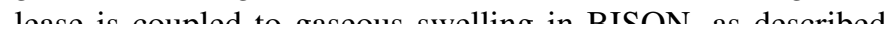

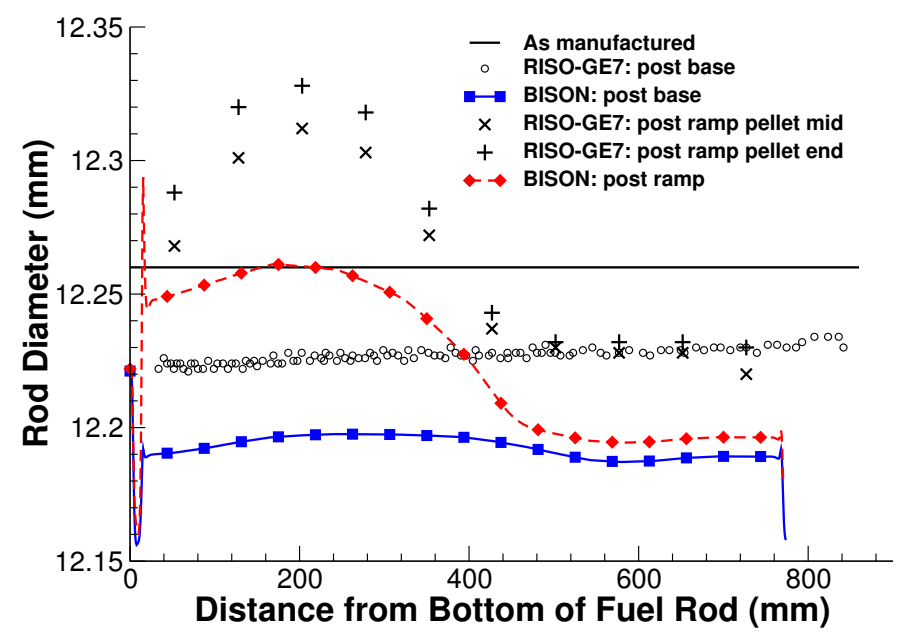

Figure 19: Comparison of the computed and measured rod diameter, as a function of rod length, for the Ris $\varnothing-3$ GE7 experiment.

Note that the Risø-3 GE7 validation case is considered in greater detail in a recent publication [51]. There the fuel is considered as either a smeared column or as discrete pellets and it is concluded that the final diameter comparison improves when considering the discrete pellet geometry. In light of this observation, a future improvement to the BISON validation effort will be the inclusion of discrete pellet analysis where appropriate.

\section{Conclusions}

BISON is a next-generation multidimensional fuel performance code that has been under development at INL since 2009. Code development is accompanied by essential code and solution verification processes. Although designed as a multi-fuels code, to date BISON has been primarily applied to LWR fuel. In this validation study, BISON predictions have been compared to 35 LWR fuel rod experiments. Results demonstrate:

- Fuel centerline temperature comparisons through all phases of fuel life are very reasonable. Deviations between predictions and experimental data are shown to be within $\pm 10 \%$ for early life through high burnup fuel and only slightly out of these bounds for power ramp experiments. Power ramp experiments generally involve a fuel refabrication process which adds to modeling uncertainty.

- Accuracy in predicting FGR appears to be consistent with state-of-the-art modeling and with the involved uncertainties.
- Comparison of rod diameter results indicate a tendency to overpredict clad diameter reduction early in life, when clad creepdown dominates, and more significantly overpredict diameter increase late in life, when fuel expansion controls the mechanical response. During power ramping, comparisons are very reasonable for low burnup fuel but grow steadily worse at higher burnups. In contrast to the base irradiation, where the radial fuel growth is overpredicted, for power ramps the measured value generally exceeds the prediction indicating the fuel diameter increase is underpredicted. Initial rod diameter comparisons are not satisfactory and have lead to consideration of additional separate effects experiments to better understand and predict clad and fuel mechanical behavior.

Results from this study are being used to define and prioritize future code development and validation activities. High priority items include:

- An increased emphasis on separate effects validation experiments, especially for fuel swelling and fuel and cladding creep.

- Inclusion of more realistic mechanical models for oxide fuel, specifically smeared cracking and creep.

- Addition of a relocation recovery model.

- Consideration of discrete pellet geometry in validation cases, especially for cladding diameter comparisons.

- Expansion of the validation base to include other fuel types including MOX and Gd-doped fuels.

- Inclusion of the effects of high burnup structure on fuel performance.

- Addition of accident behavior cases (both LOCA and RIA) to the validation base.

\section{Acknowledgments}

This work was funded by the Department of Energy Nuclear Energy Advanced Modeling and Simulation (NEAMS) program. The manuscript has been authored by a contractor of the U.S. Government under Contract DE-AC07-05ID14517. Accordingly, the U.S. Government retains a non-exclusive, royalty free license to publish or reproduce the published form of this contribution, or allow others to do so, for U.S. Government purposes.

\section{References}

[1] R. L. Williamson, J. D. Hales, S. R. Novascone, M. R. Tonks, D. R. Gaston, C. J. Permann, D. Andrs, and R. C. Martineau. Multidimensional multiphysics simulation of nuclear fuel behavior. J. Nucl. Mater., 423:149-163, 2012

[2] J. D. Hales, R. L. Williamson, S. R. Novascone, D. M. Perez, B. W. Spencer, and G. Pastore. Multidimensional multiphysics simulation of TRISO particle fuel. J. Nucl. Mater., 443:531-543, November 2013. 
[3] Pavel Medvedev. Fuel performance modeling results for representative FCRD irradiation experiments: Projected deformation in the annular AFC-3A U-10Zr fuel pins and comparison to alternative designs. Technical Report INL/EXT-12-27183 Revision 1, Idaho National Laboratory, 2012.

[4] N. N. Carlson, C. Unal, and J. D. Galloway. Formulation of the constituent distribution model implemented into the BISON framework for the analysis of performance of metallic fuels with some initial simulation results. Technical Report LA-UR-13-26824, Los Alamos National Laboratory, 2013

[5] Pavel Medvedev. Summary report on the fuel performance modeling of the AFC-2A, 2B irradiation experiments. Technical Report INL/EXT-1330006, Idaho National Laboratory, 2013.

[6] K. E. Metzger, T. W. Knight, and R. L. Williamson. Model of $\mathrm{U}_{3} \mathrm{Si}_{2}$ fuel system using BISON fuel code. In Proceedings of the International Congress on Advances in Nuclear Power Plants - ICAPP 2014, Charlotte, NC, April 6-9 2014.

[7] S.R. Novascone, B.W. Spencer, J.D. Hales, and R.L. Williamson. Evaluation of coupling approaches for thermomechanical simulations. Nuclear Engineering and Design, 295:910-921, 2015.

[8] J. D. Hales, S. R. Novascone, B. W. Spencer, R. L. Williamson, G. Pastore, and D. M. Perez. Verification of the BISON fuel performance code. Ann. Nuclear Energy, 71:81-90, September 2014.

[9] W. J. Kilgour, J. A. Turnbull, R. J. White, A. J. Bull, P. A. Jackson, and I. D. Palmer. Capabilities and validation of the ENIGMA fuel performance code. In Proceedings of the ENS Meeting on LWR Fuel Performance, Avignon, France, 1991.

[10] Fuel Analysis and Licensing Code: FALCON MOD01 - Volume 3: Verification and Validation. Technical Report EPRI-1011309, December 2004.

[11] P. Van Uffelen, A. Schubert, J. van de Laar, C. Gyori, D. Elenkov, S. Boneva, M. Georgieva, S. Georgiev, H. Hozer, D. Martens, G. Spykman, C. Hellwig, L. Nordstrom, L. Luzzi, V. Di Marcello, and L. Ott. Verification of the TRANSURANUS fuel performance code - An overview. In Proc. of the 7th International Conference on WWER Fuel Performance - Modelling and Experimental Support, Albena, Bulgaria, pages 305-320, Sep 17-21 2007.

[12] K. J. Geelhood, W. G. Luscher, and C. E. Beyer. FRAPCON-3.4: Integral Assessment. Technical Report NUREG/CR-7002 Vol.2, PNNL-19418 Vol.2, 2011.

[13] D. Gaston, C. Newman, G. Hansen, and D. Lebrun-Grandié. MOOSE: A parallel computational framework for coupled systems of nonlinear equations. Nucl. Eng. Design, 239:1768-1778, 2009

[14] D. A. Knoll and D. E. Keyes. Jacobian-free Newton-Krylov methods: a survey of approaches and applications. J. Comput. Phys., 193(2):357397, 2004.

[15] J. D. Hales, S. R. Novascone, R. L. Williamson, D. R. Gaston, and M. R. Tonks. Solving nonlinear solid mechanics problems with the Jacobian-free Newton Krylov method. CMES: Comput. Model. Eng. Sci., 84(2):123-154, 2012.

[16] J. D. Hales, R. L. Williamson, S. R. Novascone, G. Pastore, B. W. Spencer, D. S. Stafford, K. A. Gamble, D. M. Perez, R.J. Gardner, and W. Liu. BISON theory manual: The equations behind nuclear fuel analysis. Technical Report INL/EXT-13-29930, Rev.1, Idaho National Laboratory, September 2014

[17] K. Lassmann, C. O'Carroll, J. van de Laar, and C. T. Walker. The radial distribution of plutonium in high burnup $\mathrm{UO}_{2}$ fuels. J. Nucl. Materials, 208:223-231, 1994

[18] K. Lassmann, C. T. Walker, and J. van de Laar. Extension of the TRANSURANUS burnup model to heavy water reactor conditions. J. Nucl. Materials, 255:222-233, 1998.

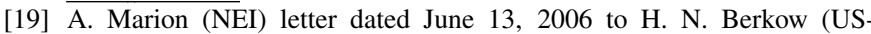
NRC/NRR). Safety Evaluation by the Office of Nuclear Reactor Regulation of Electric Power Research Institute (EPRI) Topical Report TR-1002865, Topical Report on Reactivity Initiated Accidents: Bases for RIA Fuel rod Failures and Core Coolability Criteria. http://pbadupws.nrc.gov/docs/ML0616/ML061650107.pdf, 2006.

[20] SCDAP/RELAP5-3D Code Manual. Volume 4: MATPRO a library of materials properaties for light-water-reactor accident analysis. Technical Report INEEL/EXT-02-00589, Idaho National Engineering and Environmental Laboratory, 2003.
[21] G. Pastore, L. Luzzi, V. Di Marcello, and P. Van Uffelen. Physics-based modelling of fission gas swelling and release in $\mathrm{UO}_{2}$ applied to integral fuel rod analysis. Nucl. Engrg. Design, 256:75-86, 2013

[22] G. Pastore, D. Pizzocri, J. D. Hales, S. R. Novascone, D. M. Perez, B. W. Spencer, R.L. Williamson, P. Van Uffelen, and L. Luzzi. Modelling of transient fission gas behaviour in oxide fuel and application to the BISON code. In Enlarged Halden Programme Group Meeting, Røros, Norway, September 7-12, 2014

[23] Y. Rashid, R. Dunham, and R. Montgomery. Fuel analysis and licensing code: FALCON MOD01. Technical Report EPRI 1011308, Electric Power Research Institute, December 2004.

[24] Eds. M. A. Kramman, H. R. Freeburn. Escore-the epri steady-state core reload evaluator code: General description. Technical Report EPRI NP5100, Electric Power Research Institute, February 1987.

[25] L. P. Swiler, R. L. Williamson, and D. M. Perez. Calibration of a fuel relocation model in BISON. In Proceedings of the International Conference on Mathematics and Computational Methods Applied to Nuclear Science and Engineering, Sun Valley, Idaho, May 5-9, 2013.

[26] M. Limbäck and T. Andersson. A model for analysis of the effect of final annealing on the in- and out-of-reactor creep behavior of zircaloy cladding. In Zirconium in the Nuclear Industry: Eleventh International Symposium, ASTM STP 1295, pages 448-468, 1996.

[27] D. G. Franklin. Zircaloy-4 cladding deformation during power reactor irradiation. In D. G. Franklin, editor, Zirconium in the Nuclear Industry: 15th International Symposium, pages 235-267. ASTM STP 754, 1982.

[28] M.W. Heinstein and T.A. Laursen. An algorithm for the matrix-free solution of quasistatic frictional contact problems. Internat. J. Numer. Methods Engrg., 44(9):1205-1226, 1999.

[29] A. M. Ross and R. L. Stoute. Heat transfer coefficient between $\mathrm{UO}_{2}$ and Zircaloy-2. Technical Report AECL-1552, Atomic Energy of Canada Limited, 1962.

[30] D. D. Lanning and C. R. Hann. Review of methods applicable to the calculation of gap conductance in zircaloy-clad uo2 fuel rods. Technical Report BWNL-1894, UC-78B, 1975.

[31] P. J. Roache. Verification and Validation in Computational Science and Engineering. Hermosa Publishers, Albuquerque, NM, 1998.

[32] William L. Oberkampf and Timothy G. Trucano. Verification and validation benchmarks. Nucl. Engrg. Design, 238(3):716-743, 2008. Benchmarking of CFD Codes for Application to Nuclear Reactor Safety.

[33] IAEA. Fuel Modelling at Extened Burnup (FUMEX-II): Report of a Coordinated Research Project 2002-2007. Technical Report IAEATECDOC-1687, International Atomic Energy Agency, 2002-2007.

[34] IAEA. Improvement of Computer Codes Used for Fuel Behaviour Simulation (FUMEX-III): Report of a Coordinated Research Project 20082012. Technical Report IAEA-TECDOC-1697, International Atomic Energy Agency, 2008-2012.

[35] C. R. Hann, D. D. Lanning, E. R. Bradley, R. K. Marshall, M. E. Cunningham, and R. E. Williford. Data Report for the NRC/PNL Halden Assembly IFA-432. Technical Report NUREG/CR-0560, PNL-2673, 1978.

[36] J. D. Hales, D. M. Perez, R. L. Williamson, S. R. Novascone, B. W. Spencer, and R. C. Martineau. Validation of the BISON 3D fuel performance code: Temperature comparisons for concentrically and eccentrically located fuel pellets. In Enlarged Halden Programme Group Meeting: Proceedings of the Fuels and Materials Sessions, volume HPR378, Storefjell Resort Hotel, Norway, March 10-15 2013. OECD Halden Reactor Project.

[37] E. Sartori, J. Killeen, and J. A. Turnbull. International Fuel Performance Experiments (IFPE) Database. OECD-NEA, 2010, available at http://www.oecd-nea.org/science/fuel/ifpelst.html.

[38] T. Tverberg, M. Amaya. Study of thermal behaviour of $\mathrm{UO}_{2}$ and (U,Gd) $\mathrm{O}_{2}$ to high burnup (IFA-515). Technical Report HWR-671, OECD Halden Reactor Project, 2001.

[39] P. Lösönen. Early-in-life Irradiation of IFA-562.2 (The Ultra High Burnup Experiment. Technical Report HWR-247, OECD Halden Reactor Project, 1989.

[40] K. Lassmann, A. Schubert, J. van de Laar, and P. Van Uffelen. The 'Fuel Rod Analysis ToolBox': A general program for preparing the input of a fuel rod performance code. Annals of Nuclear Energy, 81:332-335, 2015.

[41] D. M. Perez, R. L. Williamson, S. R. Novascone, R. J. Gardner, K. A. Gamble, A. T. Rice, G. Pastore, J. D. Hales, and B. W. Spencer. Assessment of BISON: A nuclear fuel performance analysis code. Technical 
Report INL/MIS-13-30314, Rev. 2, Idaho National Laboratory, September 2015 .

[42] K. Lassmann. The structure of fuel element codes. Nucl. Engrg. Design, 57:17-39, 1988.

[43] A. Bouloré, C. Struzik, and F. Gaudier. Uncertainty and sensitivity analysis of the nuclear fuel thermal behavior. Nuclear Engineering and Design, 253:200-210, 2012.

[44] The Third Risø Fission Gas Project: Bump Test AN3 (CB8-2R). Technical Report Risø-FGP3-AN3, Ris $\emptyset$, September 1990.

[45] Fuel modelling at extended burnup: Report of the Co-ordinated Research Programme on Fuel Modelling at Extended Burnup - FUMEX 19931996. Technical Report IAEA-TECDOC-998, 1998.

[46] G. Pastore, L. P. Swiler, J. D. Hales, S.R. Novascone, D. M. Perez, B. W. Spencer, L. Luzzi, P. Van Uffelen, and R. L. Williamson. Uncertainty and sensitivity analysis of fission gas behavior in engineering-scale fuel modeling. Journal of Nuclear Materials, 456:398-408, 2015.

[47] N. Cayet. Investigation of delayed fission gas release. Technical Report HWR-488, OECD Halden Reactor Project, 1996.

[48] P. Van Uffelen, A. Schubert, J. van de Laar, , and C. Györi. Development of a transient fission gas release model for TRANSURANUS. In Proc. of the Water Reactor Fuel Performance Meeting, Seoul, Korea, October 19-23, 2008.

[49] M. McGrath. In-reactor creep behaviour of Zircaloy-2 under variable loading conditions in IFA-585. Technical Report HWR-471, OECD Halden Reactor Project, 1996.

[50] K. J. Geelhood and W. G. Luscher. FRAPCON-3.5: A computer code for the calculation of steady-state, thermal-mechanical behavior of oxide fuel rods for high burnup. Technical Report NUREG/CR-7022, PNNL-19418 Vol.1, Rev 1, 2014.

[51] R. Gardner, S. R. Novascone, D. M. Perez, G. Pastore, R. L. Williamson, J. D. Hales, and W. Liu. Improving the accuracy of PCMI simulations with more realistic geometry and material models. In 2015 LWR Fuel Performance Meeting - TopFuel, Zurich, Switzerland, September 13-17 2015. 